Rev. Int. Contam. Ambie. 32 (Especial Residuos Sólidos) 91-111, 2016

DOI: 10.20937/RICA.2016.32.05.07

\title{
BIORRELLENOS: PERSPECTIVAS TRAS DOS DÉCADAS DE EXPERIENCIAS EN EL MUNDO
}

\author{
Amaya LOBO-GARCÍA DE CORTÁZAR ${ }^{1}$; María del Consuelo HERNÁNDEZ-BERRIEL ${ }^{2 *}$ y \\ María del Consuelo MAÑÓN-SALAS ${ }^{2}$
}

${ }^{1}$ Grupo de Ingeniería Ambiental. Departamento de Ciencias y Técnicas del Agua y del Medio Ambiente. Universidad de Cantabria. Av. Los Castros s/n. CP 39005, Santander, España. amaya.lobo@unican.es

${ }^{2}$ División de Estudios de Posgrado e Investigación. Instituto Tecnológico de Toluca. Av. Instituto Tecnológico s/n. Fraccionamiento La Virgen. C.P. 52149, Metepec, Estado de México, México

*Autor para correspondencia: hberriel_1999@yahoo.com y mhernandezb@toluca.tecnm.mx

(Recibido noviembre 2014; aceptado agosto 2015)

Palabras clave: relleno sostenible, pasivo ambiental, recirculación de lixiviados, biogás, biodegradación

\section{RESUMEN}

Las palabras biorrelleno, vertedero biorreactor, vertedero sostenible, resuenan con frecuencia en las reuniones técnicas y científicas de "basurólogos" desde hace tiempo. Sin embargo todavía existen muchos mitos en torno a esta técnica que, en algunos lugares, fundamentalmente de Estados Unidos, se lleva practicando durante más de veinte años. Este trabajo presenta una síntesis del estado actual del conocimiento sobre biorrellenos. Inicia revisando la historia de esta técnica, planteada por primera vez en los años 70 y aclarando sus objetivos, que son la degradación acelerada de los residuos municipales (RM), para evitar que sean o se conviertan en pasivos ambientales, así como sus ventajas e inconvenientes. Identificado el parámetro fundamental a controlar, la humedad de los RM, han surgido, según las condiciones de aireación, biorellenos anaeróbicos, semiaeróbicos, aeróbicos e híbridos. Como guía para los nuevos proyectos que se plantean y requieren en Iberoamérica, en este trabajo se reúnen las directrices generales de diseño y operación recomendables para nuevas instalaciones de biorrellenos anaeróbicos y semiaeróbicos, incluyendo los sistemas de recirculación de lixiviados y extracción del gas generado, los cambios principales respecto a la operación de un relleno tradicional y las necesidades de seguimiento. Como ejemplos se describen: una experiencia a escala real que ha servido como referencia innovadora a nivel mundial, un modelo de biorrelleno semiaeróbico aplicable a países de desarrollo intermedio y dos casos representativos del avance en este campo en Iberoamérica. Finalmente se presentan las perspectivas de avance de la técnica mediante investigación y en cuanto a su extensión en Iberoamérica.

Key words: sustainable landfill, environmental liability, leachate recirculation, biogas, biodegradation

\begin{abstract}
Concepts like 'bioreactor landfill' or 'sustainable landfill' have been resonating in specialized technical and scientific meetings for a long time. However, there are still many myths surrounding this technique which, in some places, mainly the United States, has been practiced for over twenty years. This paper summarizes the current state of knowledge on bioreactor landfills. It begins with a review of the history of this technique, proposed in the 70 s for the first time. The objective of bioreactor practices is to accelerate the degradation
\end{abstract}


of municipal waste (MW) to avoid environmental liabilities. Advantages and disadvantages of these particular landfills are discussed. Having identified the MW moisture content as the principal parameter to control, different types of bioreactors have been developed, according to the aeration modes: anaerobic, semiaerobic, aerobic and hybrid bioreactors. As a guide for new projects arising and required in Iberoamerica, general guidelines for design and operation of new anaerobic and semiaerobic installations are gathered, including the leachate reinfiltration and liquid and gas collection systems, the main operational implications, and the monitoring needs. Four practical cases are described as examples: a full scale experience which has become an international reference, a model of semiaerobic landfill suited to middle-income and developing countries, and two cases representing the progress of these methods in Iberoamerica. Finally, the prospects of advance for this practice through research and with regard to its extension in this region of the world are presented.

\section{INTRODUCCIÓN}

Las expresiones vertedero biorreactor, relleno sanitario biorreactor o biorrelleno se emplean indistintamente en trabajos y con frecuencia en reuniones técnicas y científicas de "basurólogos" desde hace más de 20 años. Todas ellas hacen referencia a un mismo concepto, que supone una evolución del relleno sanitario hacia el vertedero sostenible, para que no solamente sea un sitio de disposición final donde se controlan los impactos ambientales, sino que opere aprovechando los recursos materiales, humanos y económicos de forma armónica a lo largo del tiempo y del espacio (UNAM-FE-CECHIMEX 2012, USEPA 2007).

Sin embargo todavía existen muchos mitos en torno al vertedero biorreactor en Latinoamérica y el Caribe, España y otros países de Europa, donde aún no se ha instaurado como práctica común. Las razones de este retraso son variadas: desde la falta de personal técnico con suficiente formación y experiencia para liderar proyectos de este tipo, o el desconocimiento de esta tecnología por parte de la administración, hasta directrices políticas que se han decantado por opciones que restan interés a esta solución (OPS-AIDIIS-BID 2010). En el caso de Europa, por ejemplo, la normativa de Depósito de Residuos en Vertedero (DOE 1999) obliga a tratar los residuos municipales (RM) previamente, para recuperar materiales reciclables y reducir la materia orgánica (MO) en el relleno, disminuyendo así el riesgo contaminante de las instalaciones. En México, con la última revisión de la NOM-083-SEMARNAT-2003 se permitió la recirculación de lixiviados en las celdas de los rellenos sanitarios donde se disponen los RM, también llamados residuos sólidos urbanos (RSU), aunque no se menciona que el sitio pueda operar como biorrelleno (SEMARNAT 2003). En otros países de Latinoamérica y el Caribe ocurre algo similar (MINAM 2008).

A esto se une el recuerdo de dramáticos episodios provocados por acumulación no controlada de agua, protagonista del biorrelleno, hasta saturar los residuos: deslizamientos de grandes masas de residuo, como en Doña Juana (Bogotá, Colombia) (Hendron et al. 1999) o en Payatas (Manila, Filipinas) (Kölsch y Ziehmann, 2004) que dieron lugar a desastres ambientales y pérdidas de vidas, y que hoy se quieren evitar a toda costa.

Conforme a lo anterior es fundamental separar la opción de diseño y operación como "biorrelleno" de las prácticas de reintroducción del lixiviado o acumulación del mismo sobre la superficie del vertedero, sin otro objetivo que reducir los costes de gestión del líquido contaminado. El concepto de vertedero biorreactor se propuso por primera vez en la década de 1970, y surge con la idea de acelerar la descomposición de los RM mediante la adición de agua suplementaria a los residuos y/o la recirculación lixiviados (Pohland, 1975). Desde entonces, la Agencia de Protección Ambiental de los Estados Unidos (USEPA, por sus siglas en inglés) subvencionó diversos proyectos de prueba a distintas escalas, hasta incorporar el biorreactor como opción de operación del relleno en la regulación de vertederos, bajo el subtítulo D en la RCRA (Resource Conservation and Recovery Act) (ITRC 2006, USEPA 2007).

Al inicio, el paso a escala real de este enfoque se vio limitado por la incertidumbre respecto a la eficacia a lo largo del tiempo de las barreras de impermeabilización colocadas para evitar el escape de los lixiviados. Sin embargo, hoy en día distintos autores han comprobado que los revestimientos compuestos reducen al mínimo las fugas cuando se instalan 
correctamente (Bonaparte et al. 2002, Foose et al. 2001). En consecuencia, la introducción de agua y / o la recirculación de lixiviados es ahora plausible e incluso, desde la perspectiva del biorrelleno, deseable (Reinhart et al. 2002). Desde entonces, durante más de 20 años se han acumulado experiencias a escala real en algunos lugares, como Norteamérica o Japón, y en otros se está extendiendo rápidamente, como es el caso de Francia (EC 2009), y de países asiáticos como Malasia (Matsufuji 2004).

En este trabajo se revisa de manera resumida el conocimiento desarrollado hasta el momento en el mundo de los biorrellenos, reuniendo la información más relevante para afrontar proyectos de este tipo en países de Iberoamérica. En el siguiente apartado se describen los fundamentos básicos de estas técnicas y se muestran las tipologías alternativas. Seguidamente se reúnen las directrices generales de diseño y operación de los biorrellenos anaeróbicos y semiaeróbicos, las dos técnicas con más proyección actualmente en esta zona del planeta; así como los principales impactos a supervisar en su operación. A continuación, como ejemplo, se describen algunos casos de aplicación de las mismas y, finalmente, se comentan las perspectivas de evolución de los biorrellenos en los próximos años.

\section{FUNDAMENTOS}

\section{La importancia de la humedad}

Al contener gran cantidad de materia biodegradable, los RM depositados en vertedero son descompuestos naturalmente por microorganismos (fundamentalmente bacterias), que dan lugar a contaminación disuelta inicialmente en el lixiviado y conversión de ésta en biogás como producto final. Estos procesos son inicialmente aeróbicos, y anaeróbicos una vez agotado el oxígeno entre los poros del residuo enterrado (Sang et al. 2012). Un factor fundamental en estas reacciones es el agua. Numerosos autores han observado cómo la velocidad de degradación crece drásticamente al aumentar el contenido de humedad del residuo (Benbelkacem et al. 2010, Chugh et al. 1998, Hernández-Berriel et al. 2010, Olay-Romero et al. 2014). Por eso la técnica clásica de clausura de vertederos aislando el residuo con capas impermeables en superficie consigue no sólo reducir la cantidad de lixiviados sino también parar la generación de gas, al detener la biodegradación dejando seco el vertedero. Sin embargo esta solución es temporal, porque sólo pospone la contaminación hasta el momento en que el agua entre de nuevo en contacto con los RM, al degradarse por ejemplo la cobertura en el tiempo. Por eso surge la técnica del biorreactor.

La Asociación de Residuos Sólidos de Norte América (SWANA, por sus siglas en inglés) define como Biorrelleno un relleno sanitario (RESA) o celda del RESA donde se manejan activamente las condiciones de líquido y gas para controlar y mejorar los procesos microbianos, con el objetivo de estabilizar los constituyentes orgánicos dentro de los 5 a 10 años siguientes a su clausura (Foth y Van Dyke and Associates, Inc. 2004). "Estabilizar" involucra reducir el potencial de contaminación del vertedero en su entorno hasta hacerlo prácticamente nulo, lo que implica que el sitio de disposición no requiere más monitoreo o mantenimiento (Boda 2002, Chadetrik y Arabinda 2010). Se trata de acelerar las reacciones que ocurrirían de manera natural, para reducir el impacto de las mismas y, sobre todo, acortar en el tiempo el riesgo ambiental que supone la acumulación de residuos para, finalmente, hacer estas instalaciones más sostenibles (Hall et al. 2007, Reinhart et al. 2012, Visvanathan et al. 2010).

\section{Tipos de biorrellenos}

Con estos objetivos, y tratando de favorecer distintas reacciones en los RM, surgen los distintos tipos de biorrellenos que se plantean hoy en el mundo (ITRC 2006, SAIC 2011, Townsend et al. 2008).

\section{Biorrelleno anaerobio}

Es la técnica más extendida en occidente, consiste en añadir agua al residuo mediante recirculación de los lixiviados o/y otras fuentes, buscando alcanzar los niveles óptimos de humedad para su degradación biológica. Ésta ocurre en ausencia de oxígeno y da lugar a gas de vertedero o "biogás", principalmente compuesto por dióxido de carbono $\left(\mathrm{CO}_{2}\right)$ y metano $\left(\mathrm{CH}_{4}\right)$. Este gas ha de ser captado y/o tratado porque tiene un marcado efecto invernadero. Por otro lado, tiene un importante potencial energético que puede ser aprovechado en generación de electricidad o calor, durante los años de mayor producción.

\section{Biorrelleno semiaeróbico}

Es una técnica en expansión en distintos países asiáticos, con una tradición de más de 25 años (Chong et al. 2005, Matsufuji 2004). Se intenta acelerar la degradación del residuo en condiciones aeróbicas favoreciendo la aireación natural del vertedero a través de las conducciones de recolección de lixiviados. Para ello se instala un gran tubo central perforado conectado a tuberías laterales, todos con capacidad 
suficiente para dejar libre parte de la sección en pleno funcionamiento. En las conexiones y al final de cada tubería lateral se construyen pozos verticales de venteo hasta la superficie del vertedero. Tanto los tubos de recolección como estos pozos quedan rodeados de árido de alta permeabilidad (grava) para facilitar la circulación del aire. La diferencia de temperaturas entre el residuo (calentado por las reacciones de degradación) y el exterior provoca la introducción natural del aire a través de los conductos de lixiviado, y la emisión de los gases generados por los pozos de venteo. Al no inyectar aire forzado, no se consigue que el oxígeno penetre en todo el residuo, pero los costes de explotación son mucho menores que en el biorrelleno aerobio.

\section{Biorrelleno aerobio}

A la adición de líquido, habitualmente por recirculación de lixiviado, se añade la inyección de aire al interior del vertedero, suministrando así oxígeno para la degradación aeróbica del residuo, que es más rápida que la anaeróbica. Se generan entonces procesos similares a los de compostaje, con gran producción de calor, que puede ser aprovechado para potenciar la estabilización del vertedero en zonas frías. En esta técnica se deben controlar estrechamente las condiciones de humedad, pues las reacciones exotérmicas pueden provocar incendios por combustión de los residuos. Hoy en día se plantea como la solución más directa para estabilizar vertederos que han agotado la etapa de aprovechamiento potencial de $\mathrm{CH}_{4}$. Existen distintas estrategias de aireación, divididas en dos grandes grupos según la presión del aire: aireación a alta presión (o choques de presión) y aireación a baja presión. En esta última técnica se incluyen los sistemas de aireación activa con o sin extracción de los gases generados y la ventilación pasiva, que es la tipología descrita en el párrafo anterior. De estos sistemas se cuenta ya con experiencias a escala real en Europa y Estados Unidos (Ritzkowski y Stegmann 2012).

\section{Biorrelleno hibrido}

Surge de la combinación de las técnicas de biorrelleno anaerobio y aerobio. Los residuos de las zonas superiores, más recientemente depositados, se airean intermitentemente para acelerar la degradación de la fracción orgánica. Mientras, se intenta aprovechar también la formación de biogás en condiciones anaeróbicas en el residuo más enterrado. La secuencia fase aeróbica/anaeróbica puede seguir distintos patrones y promueve un inicio más temprano de la metanogénesis en comparación con el relleno típico "tumba seca", donde no se añaden líquidos (ITRC
2006, Kraemer et al. 2012, Kumar et al. 2011).

Sea cual sea el tipo de operación, el concepto biorrelleno puede surgir al proyectar un nuevo depósito de residuos, lo que facilita la implantación de la tecnología, o como mejora de instalaciones ya existentes, en operación o ya completas. El primero aparece especialmente en esquemas de "gestión sostenible de materiales" (Rogoff 2013), mientras que el segundo caso ha sido el más común, lógicamente, en los primeros años de implantación y desarrollo de estas técnicas (Bareither et al. 2010, YCPW 2006).

\section{Ventajas e inconvenientes}

Gran parte de estas prácticas llevan aparejados mayores esfuerzos de inversión y una operación más compleja que los rellenos sanitarios tradicionales, pero también grandes beneficios sobre la explotación, además de la reducción del riesgo postclausura y por tanto también de los costes durante esa fase (Berge et al. 2009, ITRC 2006, Kumar et al. 2011).

Las dificultades en la operación resultan de una mayor necesidad de control y un aumento en los riesgos derivados de incrementar el contenido de agua en los residuos, en su caso, que puede provocar inundación en algunas zonas y reducir la seguridad geotécnica (Ellis et al. 2005, Giri y Reddy 2014). Conforme lo anterior se requiere contar con personal cualificado, capaz de controlar los niveles de humedad de los residuos, supervisar la generación de biogás y con formación en prevención y actuación en casos de incendios y derrumbes (EA 2009, SCS ENGINEERS 2011, USEPA 2007).

Uno de los beneficios más señalados es la aceleración del asentamiento de los RM como consecuencia de una degradación más rápida y del efecto propio del paso del líquido a través de éstos. La densidad de los RM dispuestos aumenta, liberando así espacio del vertedero y prolongando la vida útil del lugar. Adicionalmente, al producirse antes el asentamiento, se evitan daños posteriores en las cubiertas e instalaciones de clausura (Abichou et al. 2013, Bareither et al. 2010, Wong 2006).

Por otro lado, al concentrarse en el tiempo las emisiones gaseosas, es más sencillo captarlas. En los biorrellenos anaerobios esto permite una mayor eficiencia en el aprovechamiento del $\mathrm{CH}_{4}$ generado al degradarse la MO, y en todo caso se traduce en un mayor control para reducción de las emisiones de efecto invernadero (Barlaz et al. 1990, Barlaz et al. 2002).

Finalmente, al reintroducir los lixiviados al vertedero, se favorece su tratamiento in situ, con el consiguiente ahorro en la gestión por reducción 
de la carga orgánica. Además, resultado de la redistribución entre los RM y otros efectos que dependen del sistema de inyección, el volumen de lixiviado recogido desciende también. Sin embargo, según las condiciones de operación, la recirculación puede provocar la acumulación de ácidos orgánicos volátiles y nitrógeno amoniacal hasta alcanzar concentraciones potencialmente inhibidoras de los procesos buscados en el vertedero (Aziz et al. 2010, Barlaz et al. 2010, Benson et al. 2007, Pohland y Kim 1999).

\section{DIRECTRICES GENERALES DE DISEÑO Y OPERACIÓN}

Como cualquier instalación para el tratamiento o disposición final de RM, el diseño y explotación de los biorrellenos debe incluir medidas para proteger la salud humana y el entorno de los posibles impactos causados por los mismos. Así, no puede concebirse un biorrelleno en un lugar sin sistema de impermeabilización que evite la migración de líquidos o gases al terreno circundante, sistema de recolección, transporte y tratamiento de los lixiviados, elementos para desvío de las aguas externas y gestión adecuada de la escorrentía sobre la superficie, incluidas coberturas intermedias y final, instalaciones para la extracción controlada y tratamiento de los gases generados con o sin aprovechamiento de los mismos. En un biorrelleno deben considerarse, además de los elementos de un relleno convencional, instalaciones y estrategias para inyectar líquidos y, en su caso, aire. Por otro lado hay que tener en cuenta la necesidad de gestionar un mayor volumen de lixiviados y biogás y un mayor riesgo de inestabilidad geotécnica, derivada de los procesos potenciados.

En los siguientes párrafos se abordan las particularidades a considerar en el diseño y operación de los biorrellenos anaeróbicos con recirculación de lixiviados, que es la tecnología más conocida, y la de mayor interés en una primera etapa de avance hacia el relleno sostenible, por sus ventajas asociadas en la gestión del lixiviado y potencial aprovechamiento del biogás. Además se describen los aspectos principales de diseño de los rellenos semiaeróbicos, como alternativa a los anteriores, cuando se busca la estabilización del relleno con menores costes.

\section{Recirculación de lixiviados \\ Instalaciones para la recirculación}

Para recircular el lixiviado generado de una manera controlada son necesarios elementos de almacenamiento, transporte y reintroducción del líquido en los RM. Como almacenamiento pueden utilizarse balsas similares a las empleadas habitualmente para regulación de los lixiviados en rellenos convencionales. Su capacidad debe ser suficiente para acumular el lixiviado durante las épocas en que el clima no permite su reintroducción. El volumen depende de la pluviometría local y la cantidad de lixiviado esperado, así como de las estrategias de operación previstas. El transporte puede realizarse por bombeo, lo que requiere una mayor inversión de instalación para equipos de bombeo, válvulas y tendido de tuberías, o en camiones cisterna. Para reducir el riesgo de atascamiento de los sistemas de inyección conviene minimizar la cantidad de sólidos recirculados, bien favoreciendo la sedimentación en las balsas de almacenamiento o simplemente evitando el bombeo de los sólidos con el agua. Finalmente, la aplicación del lixiviado puede realizarse superficialmente, o a través de conductos insertados entre los RM, verticales u horizontales. El cuadro I reúne las ventajas e inconvenientes principales de cada tipo de sistema, que se describe a continuación.

\section{a) Aplicación Superficial}

El lixiviado puede introducirse en el vertedero por aplicación directa en su superficie, a través del frente de vertido, irrigación superficial o mediante pequeñas lagunas de infiltración (menores que $6 \mathrm{~m}^{2}$, para evitar problemas de olores e insectos). En estos casos una parte significativa del líquido puede evaporarse. En estos métodos la capacidad de infiltración superficial es limitada y por ello, cuando se utilizan, suelen emplearse como complemento de otro subsuperficial. Por otro lado, en esta práctica el grado de saturación en superficie es muy elevado, lo que puede complicar la explotación el movimiento de camiones, la extensión y compactación de los residuos y, sobre todo cuando se emplean lagunas superficiales, comprometer la estabilidad del relleno (EA 2009, ITRC 2006).

\section{b) Pozos verticales}

Es la configuración más indicada para aplicar la tecnología en rellenos ya completados. Para la reinyección de los lixiviados se pueden emplear pozos verticales similares a los de extracción del biogás de vertedero. En algún caso incluso se han utilizado éstos últimos, alternando ambos cometidos. El líquido se introduce en un tubo perforado cegado en el fondo situado en el eje central del pozo, rodeado habitualmente de material granular que facilite la distribución homogénea hacia el residuo. Se han empleado tubos de acero de 2" directamente insertados 
CUADRO I. VENTAJAS E INCONVENIENTES DE SISTEMAS DE RECIRCULACIÓN DE LIXIVIADOS

\begin{tabular}{|c|c|c|}
\hline Sistema de recirculación & Ventajas & Desventajas \\
\hline $\begin{array}{l}\text { Aplicación } \\
\text { Superficial }\end{array}$ & $\begin{array}{l}\text { Fácil operación } \\
\text { Sin necesidad de instalaciones específicas }\end{array}$ & $\begin{array}{l}\text { Caudales muy limitados de infiltración } \\
\text { Riesgo de contaminación de la escorrentía } \\
\text { Riesgo de formación de aerosoles y exposición de } \\
\text { los trabajadores } \\
\text { Emisión de gases desde el lixiviado y la superficie } \\
\text { expuesta } \\
\text { Riesgo de encharcamiento de la superficie y de } \\
\text { deslizamiento }\end{array}$ \\
\hline Inyección vertical & $\begin{array}{l}\text { Fácil acceso en profundidad a celdas rellenas } \\
\text { (completadas) } \\
\text { Aplicable en celdas con coberturas intermedias de } \\
\text { baja permeabilidad } \\
\text { Operación menos afectada por asentamientos o } \\
\text { deformaciones } \\
\text { Posible utilización de los mismos conductos para } \\
\text { inyección de líquido y extracción de gas }\end{array}$ & $\begin{array}{l}\text { Caudales limitados } \\
\text { Área de influencia reducida (menor que } 10 \mathrm{~m}^{2} \text { ) } \\
\text { Escasa efectividad en altura (reparto fundamental- } \\
\text { mente en el fondo) }\end{array}$ \\
\hline Inyección horizontal & $\begin{array}{l}\text { Admite mayores caudales } \\
\text { Mayor superficie de distribución (toda la longitud) } \\
\text { Mejor reparto (mayor área de influencia) } \\
\text { Posible utilización de los mismos conductos para } \\
\text { inyección de líquido y extracción de gas }\end{array}$ & $\begin{array}{l}\text { Riesgo de deformación y rotura por aplastamiento o } \\
\text { asentamientos } \\
\text { Necesario gran caudal, suficiente para que los tubos } \\
\text { trabajen en carga } \\
\text { Mayor riesgo de atascamiento }\end{array}$ \\
\hline
\end{tabular}

Adaptado de ADEME y FNADE (2007), Benson et al. (2007), Bouchez et al. (2005), ITRC (2006).

entre el residuo y, en otros casos, pozos de hasta $1.2 \mathrm{~m}$ de diámetro, en los que se incorporan tubos normalmente de policloruro de vinilo (PVC) de $2 \mathrm{o}$ 3 ". El sistema constructivo es diferente según se trate de un relleno cerrado, donde habría que perforar con distintos métodos según el diámetro de los pozos, o un relleno en operación, donde se irán añadiendo tramos verticales a cada pozo al crecer la altura de residuo. Tanto el alcance como el volumen de inyección en este sistema es limitado, en comparación con los sistemas horizontales: en pozos de diámetro 2.5 " el rango de caudales que se ha empleado es de 220 a $570 \mathrm{~L} / \mathrm{h}$, mientras que en los pozos mayores, de $1.2 \mathrm{~m}$, se ha llegado a los $45 \mathrm{~m}^{3} / \mathrm{h}$ (ITRC 2006, Reinhart et al. 2002).

\section{c) Inyección horizontal}

Cuando el vertedero es de nueva construcción es preferible la recirculación horizontal, que permite una mejor distribución del líquido entre el residuo (Benson et al. 2007). Para ello se emplean habitualmente tubos de polietileno de alta densidad (PEAD) perforados (normalmente dos perforaciones de 6 a 12 $\mathrm{mm}$ por metro) (Townsend et al. 2008) rodeados de material de alta permeabilidad como grava u otros materiales alternativos - neumáticos, vidrio triturado o chips de madera -, insertados en zanjas entre los RM o en "mantos" (capas homogéneas de 20 a $30 \mathrm{~cm}$ de espesor de este tipo de materiales). Los tubos se prolongan cubriendo distancias de hasta $100 \mathrm{~m}$, con densidad de perforación variable para asegurar un caudal homogéneo en toda su longitud. La separación entre tubos, que debe ser lo suficientemente reducida para asegurar la humectación de todo el residuo, depende de la permeabilidad del medio. En tuberías colocadas en manto permeable puede llegar a ser de $30 \mathrm{~m}$, mientras que en el sistema de zanjas es menor; se han observado radios de influencia por debajo de $\operatorname{los} 7 \mathrm{~m}$, lo que lleva a separaciones máximas de $14 \mathrm{~m}$ (ADEME y FNADE 2007). Cada $10 \mathrm{~m}$ de altura de residuo aproximadamente se repite el sistema de distribución (con varias tuberías en cada altura) hasta llegar a cubrir todo el vertedero con varias líneas, que se suelen activar alternativamente, jugando con la apertura y cierre de válvulas en derivaciones de un tubo distribuidor común.

También en la configuración horizontal existen algunas experiencias de utilización de los mismos conductos para inyección de líquido y extracción de gas. En estos casos la operación debe ser especialmente cuidadosa al alternar recirculación de líquido, reposo y extracción de gas. Una solución más sencilla de cara a la explotación, es aprovechar la misma zanja para colocar dos tubos similares: el inferior para reinyección de líquido y el superior para captación del gas (ITRC 2006). 
Operaciones de recirculación

El objetivo es acercar la humedad del residuo a las condiciones óptimas para su degradación pero, por otra parte, se debe limitar la acumulación de lixiviado en el fondo del vertedero para minimizar el riesgo de filtraciones. La cantidad total de líquido a añadir depende de la humedad inicial y la capacidad de campo del residuo, que es la máxima humedad que éste es capaz de retener cuando drena libremente. Con estos condicionantes, el volumen a añadir suele ser muy elevado. Para residuos con humedad inicial del $25 \%$, por ejemplo, habría que añadir $150 \mathrm{~L} / \mathrm{t}$ para alcanzar el $40 \%$ de humedad, que es un valor orientativo de la capacidad de campo (ITRC 2006). Y esto suponiendo que los RM se humectan homogéneamente al introducir el líquido. La heterogeneidad de los $\mathrm{RM}$, entre otros factores, hace que la distribución en realidad sea irregular, y por eso es conveniente inyectar más líquido que el teóricamente necesario, confiando en que la circulación amplíe el alcance del sistema. Mantener el paso de líquido presenta además otras ventajas: mejora la mezcla de nutrientes en toda el área de acción, permite diluir posibles sustancias inhibidoras o inocular zonas poco activas. Por eso se plantea, en biorrellenos más avanzados, la adición de otros líquidos además del propio lixiviado (fangos de depuradora, aguas residuales industriales, aguas de escorrentía (Reinhart et al. 2002, Sandip et al. 2012, Viste 1997).

En cualquier caso se deben alternar fases de recirculación con períodos de descanso, para evitar la saturación y la formación de caminos preferenciales en los RM, y permitir la salida del gas que se va acumulando (McCreanor 1998). Lo más habitual es recircular semanal o hasta mensualmente en cada zona.

El principal parámetro de operación del sistema es el caudal de inyección, que debe permitir una distribución lo más homogénea posible. Vendrá dado por la capacidad del sistema de distribución: permeabilidad de los materiales, pérdidas de carga y presión en las conducciones. Para un mismo volumen de inyección, se consigue mejor distribución horizontal con caudales mayores y menores frecuencias de inyección. La estrategia de inyección debe contrastarse in situ, por ensayo y error de los planes iniciales. En los casos conocidos hasta el momento se han utilizado estrategias muy diferentes, con volúmenes de aplicación que varían desde 40 hasta $600 \mathrm{~L} / \mathrm{T} / \mathrm{año}$ (ADEME y FNADE 2007). En vertederos cerrados, se han utilizado tasas de aplicación por unidad de superficie entre los $5 \mathrm{~m}^{3} / \mathrm{d} / \mathrm{Ha}$ para RM muy compactados $\left(1200 \mathrm{~kg} / \mathrm{m}^{3}\right)$ y $25 \mathrm{~m}^{3} / \mathrm{d} / \mathrm{Ha}$ para $\mathrm{RM}$ más sueltos $\left(750 \mathrm{~kg} / \mathrm{m}^{3}\right)$ (ITRC 2006).
La inyección puede realizarse por gravedad o presión, aplicada mediante bombeo o altura de líquido, para mejorar la distribución y prevenir el atascamiento. Es habitual aplicar presiones bajas, del orden de 0.2 bar en el extremo de distribución (ADEME y FNADE 2007), porque presiones mayores pueden dar lugar a caminos preferenciales.

A lo largo de la operación se debe vigilar la calidad del lixiviado. Si se alcanzan $\mathrm{pH}$ por debajo de cinco debería interrumpirse la reinyección, para evitar inhibir la degradación. Varios autores han estudiado la conveniencia de pretratar el lixiviado para mejorar el proceso (Zhou et al. 2012), pero la conclusión hasta el momento es que no llega a ser necesario (ITRC 2006).

\section{Elementos del biorrelleno anaeróbico \\ Sistema de recolección de lixiviados}

Puesto que no todo el lixiviado reintroducido queda retenido entre los RM, el fondo del biorrelleno recibe mayor cantidad de líquido que un relleno convencional. En cualquier caso se debe minimizar la altura de líquido sobre el fondo, la cual por normativa se suele limitar a un máximo de $30 \mathrm{~cm}$, evitando reduciendo así el riesgo de filtraciones al exterior. En biorrellenos, esto puede obligar a aumentar las dimensiones de los conductos, que preferiblemente serán de polietileno de alta densidad (PEAD) (por resistencia y durabilidad), y si se extraen por bombeo, aumentará también la capacidad de impulsión. Hay autores que recomiendan que el sistema de extracción de lixiviados sea por gravedad, para no confiar al bombeo esta labor (Townsend et al. 2008).

Tanto en el diseño como en la operación se debe tener en cuenta un mayor riesgo de atascamiento de los conductos por precipitación química y/o crecimiento biológico a partir de los compuestos arrastrados por el lixiviado, al aumentar el paso de agua por el sistema. Un dimensionamiento holgado y el lavado frecuente de los tubos ayudarán a prevenirlo.

\section{Captación/tratamiento/utilización del biogás}

La generación de biogás durante los primeros años es significativamente mayor que en el relleno convencional, y luego la generación decrece más pronunciadamente. Por eso, para control de olores y emisiones, el sistema de captación de gas debe instalarse y ponerse en funcionamiento durante la operación del vertedero, o inmediatamente después de completada cada celda, según la configuración de la instalación. El incremento en la generación depende de la humedad original de los RM, donde la diferencia será mayor para aquellos inicialmente más secos. Se han 
llegado a observar aumentos en la tasa de generación de biogás durante los primeros años de hasta el 260 $\%$ (Augenstein et al. 2005). Lo habitual es diseñar el sistema de extracción previendo un aumento entre el 50 y el $100 \%$ en las puntas de generación, que en otro caso se convertirían en emisiones incontroladas. Para ello en los nuevos vertederos se emplean cada vez más conducciones horizontales instaladas en zanjas, aunque los típicos pozos verticales también se utilizan en otros casos.

El espaciado horizontal entre pozos en distintos lugares varía entre 10 y $60 \mathrm{~m}$, y el vertical entre conducciones horizontales entre 15 y $30 \mathrm{~m}$. El área de influencia de cada tubo se controla con la depresión del sistema de extracción. Las operaciones de extracción (juegos de apertura de válvulas, depresión en el sistema) deben ajustarse en combinación con las de recirculación, pues las dinámicas de generación y movimiento del biogás están totalmente condicionadas por el líquido introducido. Hay que prestar atención al riesgo de inundación de los conductos por lixiviados y a una mayor generación de condensados.

En todo caso el potencial de aprovechamiento del biogás, al producirse muy rápidamente, depende de los sistemas de cobertura superficial de los RM. La eficiencia en la captura puede variar entre el $35 \%$ típico para áreas en operación y el $90 \%$, cuando el residuo está cubierto con láminas impermeables. Teniendo esto en cuenta, los sistemas de aprovechamiento (motores, turbinas) deben diseñarse con flexibilidad, en vista de la gran variación en el potencial introducida al recircular.

\section{Cobertura intermedia}

Los materiales típicamente utilizados para la cobertura intermedia de los RM de baja permeabilidad, impedirían la distribución adecuada de la humedad en un biorrelleno. Por eso son preferibles materiales alternativos, como lonas provisionales que se extienden sobre el residuo y se retiran al comenzar la jornada, o lodos o espumas, formados por distintos tipos de materiales mezclados con agua, que cumplen el objetivo de cubrir provisionalmente los RM pero no quedan más tarde aislando las capas (Townsend et al. 2008). Otra opción es utilizar materiales de cobertura convencional, como minerales, que se recuperan posteriormente antes de verter la capa siguiente de RM.

En cuanto a la cobertura final las necesidades son similares a las de otros rellenos, pero a veces se pospone su instalación a la espera del asentamiento de los RM. Esto influye en la formación de lixiviados y dificulta el control de las emisiones gaseosas. Una opción cada vez más extendida es la utilización de capas biológicas permeables que favorecen la oxidación del $\mathrm{CH}_{4}$, formadas por ejemplo por $1 \mathrm{~m}$ de composta.

\section{Elementos del biorrelleno semiaeróbico}

Los RM en el biorrelleno semiaeróbico se extienden en capas uniformes de 30 a $50 \mathrm{~cm}$, hasta alturas de 2 a $3 \mathrm{~m}$. Para la cobertura diaria se recomiendan áridos minerales permeables y porosos, como arenas, en espesores de al menos $20 \mathrm{~cm}$. Este espesor debe incrementarse en caso de que los RM provoquen problemas de olor. El material de cobertura final debe alcanzar un espesor mínimo de $50 \mathrm{~cm}$. En este tipo de biorrelleno es especialmente importante que no haya acumulación de agua en la superficie, lo cual se logra con pendientes de al menos 2 a $3 \%$ (Matsufuji 2004).

Como la aireación se confía al paso de aire a través de los conductos de drenaje, éstos deben tener sección suficiente para trabajar en lámina libre, quedando al menos 2/3 del área para circulación del gas. Para el drenaje central suelen emplearse tubos de hormigón con orificios de 1" colocados entre árido seleccionado (tamaño medio entre 5 a $15 \mathrm{~cm}$ ) con conexiones laterales de tuberías de menor sección, situadas cada $2 \mathrm{~m}$. El sobredimensionamiento en la capacidad de recolección permite una evacuación muy rápida de los lixiviados, reduciendo el riesgo de contaminar el exterior por filtraciones.

El sistema de ventilación se completa con pozos construidos en cada intersección entre tubos horizontales y al final de éstos. Estos pozos, de 30 a $50 \mathrm{~cm}$ de diámetro, están formados por grava seleccionada (de 5 a $15 \mathrm{~cm}$ ), insertada en camisas cilíndricas metálicas o enmallada, e incluyen un tubo perforado de ventilación central (10 a $15 \mathrm{~cm}$ de diámetro).

El colector de lixiviados desemboca en una laguna de regulación y sedimentación. El sistema se puede completar con reinyección de lixiviados a través de los pozos de ventilación para activar reacciones y depurar el líquido antes de su tratamiento externo, por una combinación de filtración, tratamiento biológico y coagulación de sólidos.

La eficacia de los biorrellenos semi-aeróbicos se controla mediante monitoreo básico de la calidad de los lixiviados y los gases, que van revelando el proceso de estabilización (SPREP-JICA 2010). Por la sencillez del diseño y la operación, se trata de una tecnología muy adaptable a distintas condiciones locales. Tanto para la recolección de lixiviados como para los pozos de ventilación y las coberturas existen experiencias utilizando materiales específicos, que pueden encarecer el biorrelleno según su disponibilidad (hormigón, conductos plásticos, áridos de 
cantera) y otras aprovechando materiales locales, como bambú, bidones metálicos desechados, etc. (UN-HABITAT 2014a y 2014b).

\section{Principales impactos en la operación (efectos a vigilar) \\ Compactación/Asentamiento \\ La capacidad de retención y transmisión de} líquidos por el residuo disminuye con su grado de compactación. Por eso en biorrellenos no es conveniente compactar en exceso los RM. De todas maneras la degradación conlleva una pérdida de masa que, unida a la "lubricación" de las partículas con el líquido recirculado, provoca una compactación acelerada. Esto se traduce en un asentamiento significativo de la superficie en los primeros 2 a 4 años (Pohland 1975) y en consecuencia, en un aumento de la capacidad inicial de vertido. La ventaja en estos casos es el adelanto en el tiempo de estos efectos. Acelerar la degradación favorece el asentamiento durante la operación del biorrelleno, y por tanto, permite aprovechar el espacio que queda libre. El asentamiento global depende de la compactación inicial y el alcance de la degradación (Abichou et al. 2010). Pueden esperarse asentamientos entre el 20 y el $40 \%$ de la altura del biorrelleno, según las condiciones de explotación, lo que implica grandes ahorros de espacio, pero también riesgo de afectar las instalaciones del vertedero: caminos, conducciones de biogás y lixiviados, y coberturas (Bareither et al. 2010, Berge et al. 2009).

\section{Inestabilidad geotécnica}

La estabilidad geotécnica de una celda con RM depende de factores de diseño y operación. Entre los primeros destaca la pendiente de los taludes, para la que se recomienda valores máximos de $1 \mathrm{~V}: 2.5 \mathrm{H}$ (1 vertical a 2.5 horizontal) a $1 \mathrm{~V}: 4 \mathrm{H}$. Se deben considerar además las condiciones climatológicas, de manera que en lugares con alta precipitación es crucial contar con sistemas suficientes para evacuación de escorrentías y drenaje. Los mayores desastres ocurridos en vertederos de RM han estado vinculados a la acumulación incontrolada de líquido en el interior y a pendientes pronunciadas, de manera que se imparten esfuerzos en la masa y se reduce su capacidad resistente hasta causar el deslizamiento y derrumbe del relleno (Colomer et al. 2009, Colomer-Mendoza et al. 2013, Kölsch y Ziehmann 2004).

La adición de líquido en el vertedero biorreactor está precisamente encaminada a aumentar la humedad de los RM, y por tanto su grado de saturación, por lo que el riesgo de deslizamiento se incrementa si se realiza esta operación en las proximidades de los taludes: las acumulaciones locales de lixiviado en esta zona dan lugar a filtraciones laterales y pueden provocar sobrepresiones que reducen el factor de seguridad geotécnica correspondiente, por eso se aconseja no inyectar lixiviados a distancias menores que 15 a $30 \mathrm{~m}$ del borde de la pendiente exterior, usar coberturas intermedias permeables y tener previstas bombas de desagüe en la base (USEPA 2007). Por otro lado, al irse degradando con mayor rapidez la MO de los RM se producirán asentamientos diferenciales, que pueden dar lugar a esfuerzos internos añadidos y crear problemas de operación, por ejemplo al tratar de distribuir el líquido (Benson et al. 2007).

Es importante analizar la estabilidad de taludes teniendo en cuenta el grado de saturación en las situaciones de operación previstas y hacer un seguimiento detallado de los mismos (Xu et al. 2012). Hoy en día existen metodologías de análisis de estabilidad que consideran la naturaleza heterogénea y anisotrópica de los RM, y proporcionan un enfoque más fiable para el diseño y operación en vertederos biorreactores (Giri y Reddy 2014).

\section{Exfiltraciones de lixiviado en los taludes}

Las exfiltraciones de lixiviado pueden evitarse reduciendo el aporte de agua en las zonas cercanas a los taludes, alejando por ejemplo los sistemas de inyección. Por otro lado, conviene evitar capas intermedias de menor permeabilidad (coberturas) y en lo posible, cambiar el sentido habitual de la pendiente en las capas de operación, para que conduzca las aguas hacia el interior del residuo (Thiel 2005, Thiel y Monte 2005).

En cualquier caso se debe vigilar su posible aparición y cuando se detecte, evitar la migración de contaminantes con medidas como la construcción de drenes o pantallas de intercepción.

\section{Incendios}

Los riesgos de incendio se tienen tanto en tiraderos a cielo abierto como en los rellenos sanitarios. Los incendios pueden ser clasificados en superficiales y subterráneos. Los incendios superficiales ocurren en los RM que se encuentran en profundidades menores a los cinco metros, mientras que los subterráneos ocurren por debajo (EA 2007, Rim-Rukeh 2014).

Entre las causas que pueden generar un incendio superficial se tienen la disposición de residuos inflamables (plásticos, neumáticos, papel), sustancias reactivas, la emisión de biogás, el calentamiento del sol y la persistencia de altas temperaturas debido a la biodegradación aeróbica. Los incendios 
subterráneos ocurren comúnmente por el incremento de la concentración de oxígeno, que favorece la actividad bacteriana aeróbica, la cual a su vez eleva la temperatura hasta $80-90{ }^{\circ} \mathrm{C}$ y da lugar a lo que se conoce como "puntos calientes", que al contacto con bolsas de gas $\mathrm{CH}_{4}$ provocan incendios. La Agencia Ambiental (EA, por sus siglas en inglés) del Reino Unido reporta como principal factor de incendios profundos en rellenos la entrada de aire por los sistemas de extracción de biogás (EA 2007, FEMA 2002).

Aunado a lo anterior, la intrusión de aire también puede dar lugar a la creación de mezclas de gases con características explosivas, por lo que es de suma importancia controlar tanto las operaciones de extracción de biogás en los biorrellenos anaeróbicos como las de adición de aire en los biorrellenos aeróbicos (Reinhart et al. 2002, USEPA 2003). Para la extinción de incendios subterráneos se deben considerar las características de los residuos enterrados, la fuente de ignición y la profundidad del incendio. Entre las técnicas utilizadas se tiene el uso de espumas, la adición de agua o lixiviados y la inyección de nitrógeno (USEPA 2007, YCPW 2006).

\section{Seguimiento}

Un biorrelleno lleva asociado un seguimiento más exigente que otras instalaciones de vertido. Puesto que se trata de una tecnología todavía en implantación, pueden establecerse distintos niveles de monitoreo: nivel mínimo de seguimiento, si el alcance de la información a recopilar es simplemente garantizar la seguridad operativa; nivel de detalle, si se pretende optimizar el funcionamiento como biorreactor; y nivel de investigación, si la información a obtener será extrapolable a otras instalaciones.

El plan de seguimiento debe incluir datos meteorológicos (temperatura, humedad relativa, insolación, precipitación pluvial), operacionales (cantidades de RM dispuestos, condiciones de compactación, cantidad de lixiviados recogidos y recirculados, asentamientos, flujos de biogás extraído) y de caracterización de los $\mathrm{RM}$ (tipos, humedad, $\mathrm{pH}, \mathrm{MO}$, edad), del lixiviado (salinidad como indicador de la concentración de otros compuestos, demanda química de oxígeno (DQO), demanda bioquímica de oxígeno (DBO5), componentes tóxicos como metales) y del biogás (composición en pozos, emisiones). Existen varias guías que recogen listados de parámetros a controlar, incluidas frecuencias y hasta métodos recomendados (ADEME y FNADE 2007, ITRC 2006, O'Brien 2009, USEPA 2007). Para control de las operaciones en detalle es especialmente importante, a partir de los datos de seguimiento, el análisis del balance hidrológico y de la estabilización bioquímica de los RM a lo largo del tiempo.

Como complemento de los métodos tradicionales, el auge de los biorrellenos ha potenciado el desarrollo de técnicas de seguimiento detallado in situ del vertedero, como mediciones de la altura de agua sobre el fondo mediante piezómetros, o la temperatura y humedad en distintos puntos de la matriz de RM (SAIC 2011, Townsend et al. 2008, YCPW 2006).

\section{CASOS PRÁCTICOS DE REFERENCIA}

En los siguientes párrafos se describen algunos casos de biorrelleno en distintos lugares del mundo, que pueden servir de ejemplo al plantear nuevas experiencias. Se describe en primer lugar un caso pionero en Estados Unidos y de referencia a nivel mundial como biorrelleno, debido a la cantidad de información publicada sobre el mismo. Después se describen dos casos representativos del estado de implantación de estas técnicas en Iberoamérica: uno en el que se practica la recirculación de lixiviados como es habitual hoy en día en muchos lugares, previa al concepto de biorreactor, y otro que es un estudio de demostración a escala real, el primero en este ámbito geográfico con esta dimensión. Finalmente se ha incluido un biorrelleno semiaeróbico de tecnología blanda, para mostrar la otra alternativa a la gestión sostenible del relleno en países de desarrollo intermedio.

\section{EI biorrelleno del vertedero Central del Condado de Yolo (Estados Unidos)}

La experiencia más conocida entre los biorrellenos anaeróbicos en todo el mundo es la del vertedero Central del Condado de Yolo, en California (Estados Unidos). Tras varios años de experiencia piloto, en 2000 se puso en marcha un proyecto demostración dentro del programa "Project XL, eXcellence and Leadership (excelencia y liderazgo)" de la USEPA. Desde entonces se ha publicado gran cantidad de información tanto del proyecto como de sus resultados, lo que ha convertido la experiencia en modelo de referencia (Carreras 2011, USEPA 2000).

En una primera fase se construyeron tres celdas experimentales, dos anaeróbicas (CAn3.5 y CAn6) y una aeróbica (CA2.5), ocupando una extensión total de 4.86 Ha con alturas totales de $18,3 \mathrm{~m}$ en las primeras y $12.2 \mathrm{~m}$ en la tercera. El cuadro II reúne las principales características de las mismas (YCPW 2006).

Las tres celdas comparten un sistema doble de impermeabilización de fondo, y a su vez quedan separadas hidráulicamente unas de otras. El revestimiento 
de fondo se diseñó considerando la seguridad necesaria para albergar experiencias piloto. Consiste de arriba a abajo en $60 \mathrm{~cm}$ de grava como material drenante, geotextil protector, geomalla drenante, sándwich de impermeabilización compuesto por dos geomembranas de impermeabilización de 60-mil de PEAD y capa de arcilla geosintética (GCL) con permeabilidad menor que $5 \cdot 10^{-9} \mathrm{~cm} / \mathrm{s}, 60 \mathrm{~cm}$ de arcilla compactada (permeabilidad menor que $6 \cdot 10^{-9} \mathrm{~cm} / \mathrm{s}$ ), un mínimo de $15 \mathrm{~cm}$ de tierra de relleno compactada (permeabilidad menor que $10^{-8} \mathrm{~cm} / \mathrm{s}$ ), y una barrera de vapor de 40 mil de PEAD. Las tuberías para recolección de lixiviados se sitúan en zanjas, bajo la capa de grava, y conducen el líquido contaminado a los colectores que lo llevan a las lagunas de regulación. Las tres celdas cuentan con sistemas de bombeo y tuberías horizontales que permiten adicionar agua subterránea y recircular lixiviados desde las lagunas por un lado, y recuperar lixiviados de los colectores de fondo, por otro.

Como se muestra en el cuadro II, para la recolección del biogás en las celdas anaeróbicas, en las trincheras entre capa y capa se cuenta con líneas de tubería horizontal de PVC conectadas a cabezales de succión para transportar el biogás a la instalación de valorización energética del propio relleno mediante depresión. Para la operación aeróbica de la CA2.5 se utiliza un ventilador independiente, que envía el gas a un biofiltro de astillas de madera y composta para control de olores.

Además del registro continuo de flujo de gas, y entrada y salidas de líquido, para el seguimiento detallado de los procesos, cada capa de residuos de las tres celdas se equipó con sensores de temperatura, humedad, presión estática sobre el fondo y presión de poro en varios puntos. Todos los instrumentos están enlazados a un Control Automatizado de Adquisición de Datos (SCADA) con sistema de radio conectado a una computadora, para control en tiempo cercano al real. Por otro lado, se analizaron varias muestras de residuo antes de su disposición, y cada año se extraen muestras sólidas para comprobar cómo evoluciona la degradación. Además se realizan análisis periódicos de la calidad de los lixiviados y el biogás.

En el cuadro III se reportan algunos de los resultados del seguimiento de la operación durante los primeros meses, hasta 2005. Entre los beneficios más destacados se observó que en menos de tres años los lixiviados drenados alcanzaron valores de DBO5/DQO por debajo de 0.1, lo que indica su rápida estabilización. Las CAn3.5 y CAn6 además registraron una rápida disminución del contenido de metales pesados en el lixiviado. Por otro lado, tres meses después del comienzo de la adición de lixiviados se obtuvieron ya concentraciones de $\mathrm{CH}_{4}$ en el biogás de 45-55 \% v/v, y se observó una aceleración en la descomposición con aumentos en la tasa de recuperación de $\mathrm{CH}_{4}$ de 4 (CAn6) a 7 (CAn3.5) veces en comparación a las obtenidas en un RESA de control (seco). La producción de $\mathrm{CH}_{4}$ en la CAn6 de 2217 millones de $\mathrm{m}^{3}$ en 34 meses, $16 \mathrm{~m}^{3} / \mathrm{t}$ RMseco, a una tasa media de $5.6 \mathrm{~m}^{3} / \mathrm{t} / \mathrm{año}$, mientras que para un RESA seco fue de $4.4 \mathrm{~m}^{3} / \mathrm{t}$ en un periodo similar, de acuerdo al Modelo de generación de la EPA (MGB-EPA). Para la CAn3.5 se registraron 2195 millones de $\mathrm{m}^{3}$ en 29 meses lo que supone $42 \mathrm{~m}^{3} / \mathrm{t}$ RMseco, o tasas medias de $174 \mathrm{~m}^{3} / \mathrm{t} / \mathrm{año}$, muy por encima de las tasas medias de extracción en vertederos secos de hasta $3 \mathrm{~m}^{3} / \mathrm{t} / \mathrm{año}$, según la Asociación Internacional de Residuos Sólidos (ISWA, por sus siglas en inglés) ó $6.2 \mathrm{~m}^{3} / \mathrm{t}$ RM seco con el MGBEPA en un periodo similar. Además se observó una disminución en la concentración de gran parte de los contaminantes volátiles, especialmente benceno y hexano (YCPW 2006).

Los problemas operacionales encontrados en relación con la reinyección de líquidos incluyen filtraciones laterales y variaciones significativas en el contenido de humedad entre algunos puntos de las celdas más grandes, que se atribuyen a la distribución irregular provocada por partes de suelo de cobertura menos permeable.

En cuanto a costos de inversión, el total aproximado fue de 4.5 US\$/t de RM, que incluía los costos de impermeabilización de fondo y superficial, de bombeo y adición de líquido, de recuperación de biogás, de instrumentación y otros (diseño y administrativos). Basada en esta experiencia, la empresa explotadora Waste Management, Inc., reporta un costo de generación de energía entre 0.03 y $0.045 \$ / \mathrm{kWh}$.

Actualmente la mayoría de los estados de los Estados Unidos aprueban la recirculación de lixiviados en RESA con recubrimiento bajo el Subtítulo D de la RCRA y, como se ha mencionado, la USEPA impulsa desde 1995 el "Project XL" en este campo. En Australia, Alemania, Francia, Canadá y otros países desarrollados también se han impulsado experiencias sobre biorreactores, aunque no tan abundantes (Barlaz et al. 2010, Benbelkacem et al. 2010, Reinhart et al. 2002, USEPA 2007, Warith, et al. 2005).

Fruto del Proyecto XL, existe disponible información detallada como ésta de otras experiencias de explotación a escala real de biorrellenos anaeróbicos en Estados Unidos, que constituye una buena referencia al plantear proyectos de este tipo. Por otro lado, con la información generada en estas experiencias, se han 


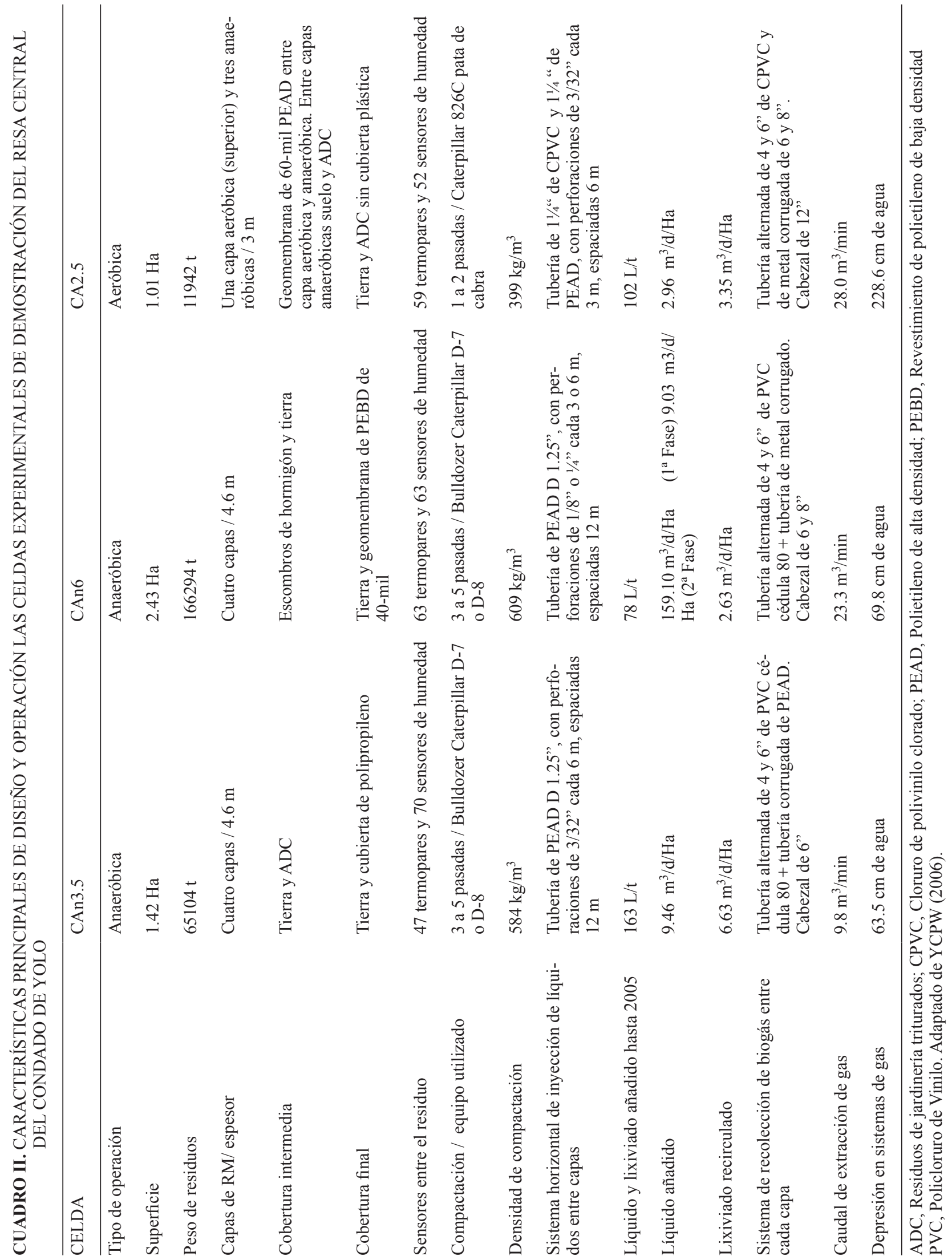




\begin{tabular}{|c|c|c|c|}
\hline CELDA & CAn3.5 & CAn6 & CA2.5 \\
\hline \multirow[t]{2}{*}{ Humedad media en RM } & $18.4 \%$ en 2002 & $22.0 \%$ en 2002 & $18.8 \%$ en 2002 \\
\hline & $40.8 \%$ en 2004 & $27.4 \%$ en 2004 & $25.7 \%$ en 2004 \\
\hline Temperatura interna de RM & 40 y $50^{\circ} \mathrm{C}$ & 40 y $50^{\circ} \mathrm{C}$ & $\begin{array}{l}\text { Se añadió agua y nitróge- } \\
\text { no líquido para reducirla }\end{array}$ \\
\hline $\begin{array}{l}\text { Asentamiento medio en } \\
2005\end{array}$ & $8.5 \%$ & $4.0 \%$ & $8.24 \%$ \\
\hline DBO5/DQO de lixiviados & 0.02 & 0.12 & 0.06 \\
\hline $\begin{array}{l}\text { Reducción de volumen de } \\
\text { celda }\end{array}$ & $\begin{array}{l}2.78 \% \text { el primer año } \\
6.45 \% \text { el segundo año }\end{array}$ & $2.75 \%$ el primer año & $\begin{array}{l}6.62 \% \text { el primer año } \\
8.24 \% \text { el segundo año }\end{array}$ \\
\hline Producción de $\mathrm{CH}_{4}$ & 42 L/kg RMsecos. & 16 L/kg RMsecos. & No reportado \\
\hline $\begin{array}{l}\text { Emisiones de } \mathrm{CH}_{4} \text { en } \\
\text { superficie }\end{array}$ & $0.8 \mathrm{ppm}$ & $3.2 \mathrm{ppm}$ & No reportado \\
\hline
\end{tabular}

Adaptado de YCPW (2006).

publicado guías para el diseño y operación de estas instalaciones, como, al comienzo, Reinhart y Townsend (1997) o más adelante ITRC (2006) o ADEME y FNADE (2007) y también informes detallados con los resultados en distintos casos (Bareither et al. 2010, Barlaz et al. 2010, Benson et al. 2007, Warith et al. 2005).

\section{El caso del relleno de Huaycoloro (Perú).}

Aunque hoy en día no se hayan descrito casos de biorrellenos a gran escala en Iberoamérica, lo cierto es que en muchas instalaciones se lleva recirculando el lixiviado de una manera u otra desde hace tiempo. Ésto ha llevado a problemas graves en algunos casos, pero también a casos de éxito, como el que se describe a continuación. La diferencia entre el fracaso y la buena práctica estriba en el correcto seguimiento y control de las operaciones.

Perú como la mayoría de los países de Latino América, enfrenta grandes retos en cuanto a la gestión de los RM, debido a que la composición a nivel nacional comprende alrededor de $50.9 \%$ de $\mathrm{MO}$ y $24.8 \%$ de reciclables. Para el 2012 Perú contaba con 10 RESA autorizados, donde se dispusieron sólo el $38 \%$ de los 7.1 millones t de RM generados (MINAM 2012).

Entre estos lugares se encuentra el de Huaycoloro, que fue el primer relleno privado de Perú, operado por la empresa PETRAMÁS. Sus operaciones se iniciaron en 1994, con la recuperación de la Quebrada de Huaycoloro, que era utilizada como botadero. Actualmente recibe las 24 horas del día una media de $3500 \mathrm{t}$ de RM, que constituyen alrededor del $42 \%$ de los residuos que genera la ciudad de Lima Metropolitana.
Los RM se manejan de manera ordenada, con cobertura diaria y reintroducción de los lixiviados mediante trincheras horizontales. Esto último es una práctica permitida en el país, recogida en las guías técnicas de gestión de vertederos como estrategia para el control del lixiviado (MINAM 2008).

Inicialmente el biogás del sitio era controlado mediante quemado, hasta que en marzo de 2007 se puso en marcha un proyecto de Mecanismo de Desarrollo Limpio (MDL), para la captura y combustión del biogás generado, con objetivo de reducir en 7 años el equivalente a $2000000 \mathrm{t}$ de $\mathrm{CO}_{2}$. El sistema de captación y aprovechamiento de gas instalado incluye 200 pozos y un gaseoducto de más de $10 \mathrm{~km}$ hasta la estación de succión y quemado automatizado, que genera 4.8 MWh de energía eléctrica (PETRAMÁS 2011 y 2013).

En estas condiciones, el relleno de Huaycoloro no puede considerarse biorrelleno en los términos descritos anteriormente, pues la recirculación de lixiviados se plantea como solución para su gestión más que en busca de la estabilización de los RM. No obstante las ventajas son similares e incluyen el estímulo de la generación de biogás, optimizando su aprovechamiento. Además, sus necesidades de control se incrementan respecto a un relleno tradicional, aunque serán menores que en los biorrellenos de experimentación descritos aquí.

\section{La celda experimental de Albacete (España)}

Más allá de la opción descrita en el apartado anterior, en Iberoamérica se han desarrollado distintas experiencias para optimización del vertedero biorreactor a escala de laboratorio y piloto, como los 
estudios de México (Delgado-Hernández et al. 2013, Hernández-Berriel et al. 2012), e incluso en Chile se han llevado a cabo experiencias a escala campo (Palma et al. 2000).

Como ejemplo de las experiencias a escala real que hoy en día se están planteando en Iberoamérica, se encuentra la experiencia piloto que la empresa Ferrovial (Ferrovial Servicios Cadagua) está desarrollando desde el año 2011 en el Centro de Tratamiento de Residuos Urbanos (CTRU) de Albacete.

Este proyecto, única experiencia de este tipo hasta el momento en España, pretende la demostración a escala real de las mejores técnicas disponibles para optimizar la explotación como biorreactor de un vertedero de RM, con el objetivo final de reducir los riesgos ambientales, así como la duración del periodo post-clausura, asegurando su estabilidad una vez concluido dicho periodo. La experiencia tiene una duración prevista de 6 años y su finalización se estima para diciembre de 2017.

Para el desarrollo del proyecto se aprovechó un área del vertedero municipal en la que se ha construido una celda biorreactor que se explota en paralelo a una celda convencional (Celda control), para posibilitar la comparación de los resultados obtenidos. La celda biorreactor presenta dimensiones de 90 por $65 \mathrm{~m}$ en la base y ha sido construida en un área sobre una celda anterior, con lo que se elevará hasta $24 \mathrm{~m}$ de altura media. Sobre ella se va extendiendo y compactando los RM hasta una densidad media inicial de $0.95 \mathrm{t} / \mathrm{m}^{3}$. Se prevé el vertido en cada celda un total de $150000 \mathrm{t}$ de residuo, compuesto principalmente por residuo voluminoso, rechazos del afino de pretratamiento y del tratamiento de RM recogido mezclado y rechazo de tratamiento de la fracción de envases ligeros. Ambas celdas quedarán aisladas entre sí y del resto de los residuos mediante capas geosintéticas impermeables, sobre las que está previsto continuar el vertido una vez completadas.

El sistema de reinfiltración de lixiviados se basa en la técnica de mantos o capas permeables (Haydar y Khire 2007) instalados a varias alturas de la celda y consistente en una capa de material granular de 20 a $30 \mathrm{~cm}$ de espesor, colocado entre geotextiles que actúan de filtro. En la capa permeable se insertan las correspondientes tuberías ranuradas de infiltración. Para evitar exfiltraciones laterales, la capa permeable queda rodeada en su perímetro por un cordón de tierra. El lixiviado, procedente de la balsa del vertedero, es introducido a presión a través de estas tuberías mediante bombeo varias veces al mes.

Entre los parámetros a estudiar en la gestión del sitio se encuentran la cantidad y calidad del biogás captado. Para ello tanto en la celda biorreactor como en la celda control se han instalado tuberías de captación de biogás en forma de espinas de pez apoyadas sobre los taludes. Además, en la celda de control se han instalado pozos verticales de captación, siguiendo el modelo del resto del vertedero. En la celda biorreactor por su parte, se tiene previsto aprovechar las capas de infiltración para la captación y extracción de biogás en los periodos comprendidos entre episodios de reinfiltración.

Para cuantificar en detalle los resultados de la gestión del vertedero como biorreactor se han instalado equipos de medida y control, como por ejemplo células de presión total para el seguimiento continuo de las presiones en el interior de los residuos. Entre las acciones planificadas para el seguimiento del proyecto de investigación, se incluyen la monitorización diaria del residuo depositado, volumen de lixiviado producido, condensados en la red de biogás, variables meteorológicas y seguimiento de las operaciones de reinfiltración realizadas. Además, se realizan periódicamente análisis de lixiviados recogidos y recirculados, del biogás captado y las emisiones superficiales, caracterización del residuo y levantamiento topográfico. Complementariamente se realizarán varias tomografías de resistividad eléctrica para completar el análisis de la respuesta del vertedero a las inyecciones de lixiviado. Finalmente, al terminar el proyecto se caracterizarán varias muestras de sólido extraídas del vertedero para evaluar su estabilidad y se aprovechará para medir la temperatura en distintos puntos del interior.

Hasta el momento se han vertido aproximadamente 90000 t de residuo en cada celda, sobre las que se sitúa la primera capa de reinfiltración y ya se ha empezado a rellenar el segundo nivel (Ferrovial Servicios 2014). Las primeras operaciones de reinyección, desde otoño del 2013, han mostrado el buen funcionamiento del sistema de reinfiltración y de los equipos de seguimiento, pero a estas alturas todavía no existen resultados concluyentes para evaluar el diseño o su impacto sobre la estabilización de los residuos.

\section{El relleno de Ampang Jajar (Malasia)}

El vertedero de Ampang Jajar en el municipio de Seberang Perai (Malasia) era en 1988 un vertedero incontrolado con frecuentes incendios, problemas de olores, humos y vectores sanitarios. Las obras de adecuación de esta instalación son un ejemplo de aplicación del concepto de biorrelleno en países en desarrollo, con recursos limitados y en particular a instalaciones en operación (Raju et al. 2001) 
Para abordar el problema el lugar se dividió en varias secciones. Cada una fue impermeabilizada por separado y con sistemas de extracción de lixiviado y ventilación de biogás independientes. En la primera etapa de mejora, que duró dos años, se acotó la zona de vertido mediante caballones de separación. Para estos muros perimetrales y para la impermeabilización se aprovecharon RM antiguos, depositados al menos dos años antes en el propio vertedero, y la tierra obtenida al excavar para construir los drenes perimetrales. La tierra restante de la excavación fue aprovechada como material de cobertura intermedia al ir disponiendo los RM. Para los caminos de acceso se aprovecharon residuos de construcción y demolición. Entre los RM se construyeron los pozos de venteo de biogás mediante contenedores metálicos desechados (tambores de aceite) rellenos de grava y bambú. Además se adecuaron las vías de acceso al vertedero y se instaló una báscula para contabilizar y registrar el ingreso de los RM. También se desarrolló un plan ordenado, que redujo el frente de vertido y se estableció la cobertura diaria de los residuos para evitar incendios.

En una segunda etapa, que se prolongó otros dos años, se aplicó el concepto de vertedero semiaeróbico a las nuevas celdas. Para ello se adaptó la tecnología Fukuoka original a las limitaciones de recursos del lugar. El sistema de recolección de lixiviado se construyó con un colector central de hormigón con tubos laterales de bambú agujereados (agujeros de 3 a $5 \mathrm{~cm}$ ) cada 30 a $50 \mathrm{~cm}$. Todos los tubos se colocan rodeados de árido seleccionado (entre 5 y $15 \mathrm{~cm}$ de tamaño medio) y pendientes adecuadas. En cada punto de unión de los tubos laterales con el colector se colocó un pozo de venteo, formado por un contenedor metálico perforado (agujeros de 3 a $5 \mathrm{~cm}$ separados 30 a $50 \mathrm{~cm}$, al tresbolillo en toda la altura del pozo). Cada pozo se rellenó de grava filtrante rodeando el tubo de extracción del gas, en este caso de plástico. El lixiviado fue conducido a una pequeña laguna de recolección, dotada de un aireador para su pretratamiento. Mediante bombeo, se recirculó a través de la grava de los pozos de venteo, que actuó de filtro depurador. Con este método simplificado, además de acabar con los problemas más llamativos de molestias por olores, incendios y vectores, se obtuvieron mejoras notables en la calidad del lixiviado, que se mantuvo estable con concentraciones de DBO y DQO del orden de 400 y $2000 \mathrm{mg} / \mathrm{L}$ respectivamente, a pesar de ser un vertedero "joven" (Ramly y Manmat 2001).

El Método Fukuoka o "Aireación pasiva" ha sido aprobado como MDL. Como en el caso descrito, ha sido puesto en práctica en varias ciudades asiáticas gracias a la Agencia Japonesa de Cooperación Internacional (JICA, Japan International Cooperation Agency) y en otros países del mundo por medio del Programa de las Naciones Unidas para los asentamientos humanos (ONU-HABITAT). Entre los casos de éxito, se encuentran los rellenos semi-aeróbicos de Pulau Burung en Malasia, el de Nam Son de la ciudad de Hanio en Vietnam, que se culminó en 2005, el de la ciudad de Weifang en China, cuya construcción incluyó 6.4 Ha para 1.16 millones t de RM y finalizó en 2003 y la transformación de tiradero a biorrelleno de la isla de Samoa de Tafaigata Upolo del Pacífico (SPREP-JICA 2010, SPREP 2014, UN-HABITAT 2014a y 2014b).

\section{PERSPECTIVAS}

La extensión del concepto de biorrelleno es consecuencia lógica de la necesidad de tratamiento del líquido contaminado, y a ello lleva el progreso en las exigencias de control de la contaminación generada en los sitios de disposición final. Como se ha descrito en los apartados anteriores, hoy en día se cuenta en el mundo con experiencias a escala real de los distintos tipos de explotación de los rellenos como biorreactores, con demostración de las principales ventajas e inconvenientes de cada tecnología.

La opción mejor implantada, por su mayor sencillez y el atractivo de un mejor aprovechamiento del biogás, es la de explotación anaeróbica con recirculación de lixiviados. Es una alternativa que parece especialmente apropiada en regiones como América Latina y El Caribe, debido al gran contenido de MO en los RM y a las estrategias de gestión que llevan éstos mayoritariamente a vertedero; además de reducir el pasivo ambiental, permite un mejor control y aprovechamiento del biogás de vertedero y, por tanto, obtener un mayor rendimiento de la gestión de los RM, especialmente potenciado a partir de la instauración del mercado de bonos de carbono. De hecho, el MDL ha permitido en los últimos años financiar muchos proyectos de captura y aprovechamiento de $\mathrm{CH}_{4}$ en la región (Carreras 2013). Ésto ha despertado un interés creciente en los últimos años que se está plasmando en proyectos demostración en varios países iberoamericanos. Sin embargo en esta zona, donde apenas poco más del $50 \%$ de la población cuenta hoy en día con un sitio de disposición para sus residuos que cumpla con las mínimas condiciones sanitarias (OPS-AIDIS-BID 2010), el biorrelleno es todavía una idea emergente. 
Si bien los esfuerzos se centran en crear rellenos, un mayor conocimiento sobre la gestión del lixiviado y el interés por la recuperación de gas, junto con la incorporación de técnicos adecuadamente formados para su operación controlada, podría conducir a la proyección y consolidación de biorrellenos como forma de explotación sostenible.

A nivel mundial, aunque puede afirmarse que las técnicas están puestas a punto, queda margen para la mejora en distintos aspectos actualmente en investigación, como los que se describen en los siguientes párrafos.

Más allá del pretratamiento obligado en Europa para recuperar reciclables y reducir la MO de los RM, se pueden plantear tratamientos de éstos previos a su vertido para mejorar su degradación en el relleno. Una opción sencilla, aunque no del todo efectiva, es la trituración, para reducir el tamaño de partícula y así facilitar el acceso del agua y los microorganismos a toda la masa (Reinhart y Townsend 1997).

Como se ha comentado, en ocasiones es necesario depurar el lixiviado antes de su reintroducción, con el objetivo de reducir posibles concentraciones inhibidoras, o para evitar la acidificación del relleno. Dando la vuelta a esta necesidad, se estudia depurar el lixiviado dentro del relleno. En rellenos híbridos por ejemplo, puede aprovecharse la zona aeróbica para oxidar parte del nitrógeno amoniacal que se va concentrando en condiciones anaeróbicas, y lograr su conversión a nitrógeno gas favoreciendo distintos procesos, como la nitrificación/desnitrificación o el proceso Anammox (Berge et al. 2005). Incluso se ha planteado el intercambio de lixiviados entre celdas de vertido de distinta antigüedad con este objetivo, o el combinar el tratamiento del lixiviado recirculado con la aireación del relleno (Abichou et al. 2013, Batarseh et al. 2010).

Por otro lado, la necesidad de mayores volúmenes de líquido que los generados en el lugar ha obligado a buscar fuentes externas. Estos líquidos contribuyen además a diluir algunos componentes inorgánicos que pueden ser problemáticos si se acumulan. Entre otros, pueden emplearse aguas residuales urbanas o industriales que, añadiendo carga orgánica, aumentan el potencial de $\mathrm{CH}_{4}$, en lo que sería un co-tratamiento de residuos sólidos y líquidos. Actualmente no es extraño, por ejemplo, la introducción de fangos de depuradora, incluso en rellenos tradicionales (ISWA 1997, USEPA, 2003).

En definitiva, se buscan estrategias para hacer el relleno sostenible (Scharff 2007), es decir, para evitar que el sitio de disposición final se convierta en un almacén de contaminación que deberán resolver generaciones futuras. Con esta idea se evalúan distintas combinaciones de fases anaeróbicas / aeróbicas para la estabilización final, con la opción posterior del lavado o "flushing" (IWM 1999, Reinhart et al. 2012), para arrastrar contaminantes inorgánicos que pueden quedar inmovilizados temporalmente, pero que posteriormente lixiviarán al abandonar el vertedero, como es el caso de los metales pesados, que pueden quedar precipitados en forma de sulfuros o complejos húmicos, y luego pueden ser lavados al penetrar el oxígeno. El ciclo del sitio se cerraría haciendo minería del lugar, para recuperar los materiales, una vez descontaminados.

Como complemento imprescindible a estas directrices, diversos grupos de investigación trabajan intentando definir los criterios de estabilidad para rellenos y biorrellenos, con indicadores que permitan afirmar que el lugar ha sido descontaminado y puede ser abandonado, puesto que ya no supone un riesgo para el medio ambiente y la salud humana (Abichou et al. 2013, Barlaz et al. 2002, Decottingnies et al. 2005, Francois et al. 2006, Sri-Shalini et al. 2010, Trois y Griffth 2008).

Las opciones de explotación como biorreactor de un sitio de disposición final suponen un sobrecoste sobre la inversión inicial de acondicionamiento de un nuevo relleno, provocado por los equipos de operación para control del sistema. Sin embargo, este sobrecoste se ve compensado en grandes vertederos con los beneficios derivados del aumento de la capacidad de vertido, la mejor explotación y aprovechamiento del biogás, habitualmente para generación de electricidad, y el ahorro en tratamiento de lixiviados (Berge et al. 2009). El balance es muy sensible al escenario de precios de venta de la electricidad generada y en zonas como Iberoamérica, a la evolución del mercado de bonos de carbono, del que depende el beneficio en la venta de emisiones de $\mathrm{CO}_{2}$ evitadas como MDL. En todo caso los proyectos sí son económicamente rentables en el largo plazo, al considerar el ahorro en los costes de vigilancia postclausura (gestión de lixiviados, control de emisiones difusas, etc.) y los beneficios de adelantar el uso postclausura. Esto implica que se irán extendiendo en los distintos países a medida que la responsabilidad del período de vigilancia postclausura quede definida y sea efectiva.

\section{AGRADECIMIENTOS}

Los autores agradecen la posibilidad de realizar este trabajo conjunto entre diferentes países iberoamericanos a CYTED (Proyecto 715RT0494). 


\section{REFERENCIAS}

Abichou T., Barlaz M.A., Green R. y Hater G. (2013). The Outer Loop bioreactor: A case study of settlement monitoring and solids decomposition. Waste Manage. 33, 2035-2047. http://dx.doi.org/10.1016/j. wasman.2013.02.005

ADEME y FNADE (2007). State of the Practices and Implementation Recommendations for Non-hazardous Waste Management using Bioreactor landfills. French Federation of Waste Management Services and French Environment and Energy Management Agency. Technical coordination: Isabelle Hebe - Département Gestion Optimisée des Déchets- Direction Déchets et Sols - ADEME Angers December 2007. [en línea]. http://www.ademe.fr/sites/default/files/assets/documents/51261_bioreactor.pdf

Augenstein D., Yazdani R., Kieffer J. y Benemann J. (2005). Yolo County, California Controlled Landfill Program:A summary of results since 1994. Memorias. Sardinia 2005. Tenth International Waste Management \& Landfill Symposium. CISA, Environmental Sanitary Engineering Centre, Cagliari, Italy. 3-7 October 2005. CD-ROM.

Aziz S. Q., Aziz H. A., Yusoff M. S., Bashir M.J.K. y Umar M. (2010). Leachate characterization in semi-aerobic and anaerobic sanitary landfills: A comparative study. J. Environ. Manage. 91, 2608-2614. http://dx.doi. org/10.1016/j.jenvman.2010.07.042

Bareither C. A., Benson C. H., Barlaz M. A., Edil T. B. y Tolaymat T. M. (2010). Performance of North American bioreactor landfills I: Leachate hydrology and waste settlement. J. Environ. Eng. 136, 824-838.

Bareither C. A., Wolfe G. L., McMahon K. D. y Benson C. H. (2013). Microbial diversity and dynamics during methane production from municipal solid waste. Waste Manage. 33, 1982-1992. http://dx.doi.org/10.1016/j. wasman.2012.12.013

Barlaz M. A., Ham R. K y Schaefer D. M. (1990). Methane production for municipal refuse: a review of enhancement techniques and microbial dynamics. Crit. Rev. Environ. Control. 19, 557-584.

Barlaz M. A., Rooker A. P., Kjeldsen P., Gabr M. A. y Borden R. C. (2002). Critical Evaluation of Factors Required To Terminate the Postclosure Monitoring Period at Solid Waste Landfills. Environ. Sci. Technol. 36, 3457-3464. DOI: 10.1021/es011245u

Barlaz M. A., Bareither C. A., Hossain A., Saquing J., Mezzari I., Benson C.H., Tolaymat T.M. y Yazdani R. (2010). Performance of North American bioreactor landfills II: Chemical and biological characteristics. J. Environ. Eng. 136, 839-853. http://dx.doi.org/10.1061/ (ASCE)EE.1943-7870.0000220
Batarseh E. S., Reinhart D. R. y Berge N. D. (2010) Sustainable disposal of municipal solid waste: Post bioreactor landfill polishing. Waste Manage. 30, $2170-$ 2176. http://dx.doi.org/10.1016/j.wasman.2010.06.015

Benbelkacem H., Bayard R., Abdelhay A., Zhang Y. y Gourdon R. (2010). Effect of leachate injection modes on municipal solid waste degradation in anaerobic bioreactor. Bioresource Technol. 101, 5206-5212. http:// dx.doi.org/10.1016/j.biortech.2010.02.049

Benson C.H., Barlaz M.A., Lane D.T. y Rawe J.M. (2007). Practice review of five bioreactor/recirculation landfills. Waste Manage. 27, 13-29. http://dx.doi. org/10.1016/j.wasman.2006.04.005

Berge N.D., Reinhart D.R. y Townsend T.G. (2005). The fate of Nitrogen in Bioreactor Landfills. Crit. Rev. Env. Sci. Technol. 35, 365-399. http://dx.doi. org/10.1080/10643380590945003

Berge N.D., Reinhart D.R. y Batarseh E.S. (2009). An assessment of bioreactor landfill costs and benefits. Waste Manage. 29, 1558-1567. http://dx.doi.org/10.1016/j. wasman.2008.12.010

Boda B. (2002). Evaluation of Stability Parameters for Landfills. Master of Science Thesis. Faculty of Virginia Polytechnic Institute and State University, Blacksburg, Virginia, USA. 49 pp.

Bonaparte R., Daniel D. y Koerner R. (2002). Assessment and recommendations for improving the performance of waste. Containment Systems, Report No. EPA/600/R-02/099, US Environmental Protection Agency, Cincinnati, Oh, USA [en línea]. http://www. nrc.gov/docs/ML1217/ML12179A248.pdf 12/11/2014

Bouchez T., Duquennoi C. y Barina G. (2005). Vers une nouvelle generation de centres de stockage bioactifs. Tech. Sci. Meth.: Gen. Urbain Gen. Rural. 7-8, 66-77.

Carreras N. (2011). Producción de energía en vertederos españoles. El vertedero Biorreactor. Experiencias en EEUU. Memorias. XIII Conferencia ATEGRUS sobre vertederos controlados. Lleida, España. 19 al 20 de Octubre, 2011. CD-ROM.

Chadetrik R. y Arabinda Sh. (2010). Municipal solid waste stabilization by leachate recirculation: A case study of Ambala City. Int. J. Environ. Sci. 1, 645-655.

Chong T. L., Matsufuji Y. y Hassan M. N. (2005). Implementation of the semi-aerobic landfill system (Fukuoka method) in developing countries: a Malaysia cost analysis. Waste Manage. 25, 702-711. http://dx.doi. org/10.1016/j.wasman.2005.01.008

Chugh S., Clarke W., Pullammanappallil P. y Rudolph V. (1998). Effect of recirculated leachate volume on MSW degradation. Waste Manage. Res. 16, 564-573. DOI: $10.1177 / 0734242 X 9801600607$

Colomer F.J., Gallardo A., Bovea M.D. y Carlos M. (2009). Evaluación del riesgo geotécnico en vertederos de re- 
siduos sólidos: Identificación de los Principales Peligros. Memorias. II Simposio Iberoamericano de Ingeniería de Residuos. REDISA, Universidad del Norte. Barranquilla, Colombia. 24 al 25 de septiembre, 2009. CD-ROM. Colomer-Mendoza F. J., Esteban-Altabella J., GarcíaDarás F. y Gallardo-Izquierdo A. (2013). Influence of the Design on Slope Stability in Solid Waste Landfills. Earth SCI. 2, 31-39. http://dx.doi.org/10.11648/j. earth.20130202.12

Delgado-Hernández Ma.C., Hernández-Berriel Ma.C., Barrientos-Becerra B. y Márquez-Benavides L. (2013). Effect of Frecuency and Recirculated Volume in Produced Leachate. Proceedings the 26th International Conference on Solid Waste Technology and Management. Philadelphia, PA U.S.A. March 10-13, 2013. 11-19.

Decottignies V., Galtier L., Leffebvre X. y Villerio T. (2005). Comparison of analytical methods to determine the stability of municipal solid waste and related wastes. Memorias. Sardinia 2005, Tenth International Waste Management and Landfill Symposium. CISA, Environmental Sanitary Engineering Centre, Cagliari, Italy. 3-7 October 2005. CD-ROM.

DOE (1999). Directiva 1999/31/CE del Consejo relativa al vertido de residuos. El Consejo de la Unión Europea. Diario Oficial de las Comunidades Europeas (DOE). 26 de abril de 1999. 19pp.

EA (2007). Review and Investigation of deep-seated fires within landfill sites Science. The Environment Agency is the leading public body protecting and improving the environment in England and Wales. Report: SC010066. ISBN: 978-1-84432-681-5. (C) Environment AgencyMarch 2007. UK. 98pp

EA (2009). A technical assessment of leachate recirculation. The Environment Agency is the leading public body protecting and improving the environment in England and Wales. Report: SC030144/R6. ISBN: 978-1-84911-147-8. UK. 83pp.

EC (2009). Assessing legal compliance with and implementation of the waste acceptance criteria and procedures by the EU-15. Final Report. European Commission (EC), Brussels. [en linea]. http://ec.europa. eu/environment/waste/pdf/report_wac.pdf 5/04/2015

Ellis G.T., Park J., Debik E. y Smith S. (2005). Evaluation of Leachate Treatment and Recycle Options at the Boone County Landfill. Memorias. The Twenty International Conference on Solid Waste Technology and Management. Philadelphia, PA, U.S.A. 3-6 April 2005. CD-ROM.

FEMA (2002). Landfill Fires Their Magnitude, Characteristics, and Mitigation. May 2002/FA-225 Federal Emergency Management Agency (FEMA). United States Fire Administration. [en linea]. http://www.
sustainable-design.ie/fire/FEMA-LandfillFires.pdf 30/03/2015

Ferrovial Servicios (2014). La celda biorreactor de Albacete. Informe Interno de Seguimiento. Barcelona, España. $60 \mathrm{pp}$

Foose G., Benson C. y Edil T. (2001). Predicting leakage through composite landfill liners. J. Geotech. Geoenviron. Eng. 127, 510-520. http://dx.doi.org/10.1061/ (ASCE)1090-0241(2001)127:6(510)

Foth \& Van Dyke and Associates, Inc. (2004). Updated Research Report on Bioreactor Landfills, Landfill Leachate Recirculation and Landfills with Methane Recovery. Scope ID: 04R004. [en línea]. http://static1. squarespace.com/static/55118948e4b06b1b4f71b1f4/t/ 5613fb7fe4b0982e4b9b3118/1444150143764/PC Research_Report_on_Bioreactor_Landfills.pdf 5/11/2013

Francois V., Feuillade G., Skhiri N., Lagier T. y Matejka G. (2006). Indicating the parameters of the state of degradation of municipal solid waste. J. Hazard. Mater. 137, 1008-1015. http://dx.doi.org/10.1016/j. jhazmat.2006.03.026

Giri R.K. y Reddy K.R. (2014). Slope stability of bioreactor landfills during leachate injection: Effects of heterogeneous and anisotropic municipal solid waste conditions. Waste Manage. Res. 32, 186-197.

DOI: $10.1177 / 0734242 X 14522492$

Hall D.H., Drury D., Gronow J.R., Pollard S.J. y Smith R. (2007). Estimating Pollutant Removal Requirements for Landfills in the UK: III. Policy Analysis and Operational Implications. Environ. Technol, 28, 25-32. http://dx.doi.org/10.1080/09593332808618766

Haydar M. M. y Khire M. V. (2007). Leachate recirculation using permeable blankets in engineered landfills. J. Geotech. Geoenviron. Eng. 133, 360-371. http://dx.doi. org/10.1061/(ASCE)1090-0241(2007)133:4(360)

Hendron D. M., Fernández G., Prommer P. J. y Orozco L. F. (1999). Investigation of the cause of the 27 september 1997 slope failure at the Doña Juana Landfill. Memorias. Sardinia 1999. 7th International Waste Management and Landfill Symposium. S. Margherita di Paula, Cagliari, Italy. 4-8 October, 1999. 545-567.

Hernández-Berriel Ma.C., Mañón-Salas C., SánchezYáñez J.M., Lugo J. y Márquez-Benavides L. (2010). Influence of Recycling Different Leachate Volumes on Refuse Anaerobic Degradation. Open Waste Manage. 3, 155-166. DOI: 10.2174/1875934301003010155

Hernández-Berriel Ma. C., Álvarez Fragoso N.O., Vaca Paulín R., Márquez Benavides L. y Lugo de la Fuente J. (2012). Determinación de metales pesados en sólidos y lixiviados en biorreactores a diferentes tasas de recirculación. Rev. Int. Contam. Ambie. 28, 77-82.

ISWA. (1997). Sludge treatment and disposal. Management Approaches and Experiences. International Solid 
Waste Association (ISWA). Environmental Issues Series, Vol. 7. European Environmental Agency. Report No 77. Copenague, Dinamarca. 54 pp.

ITRC (2006). Characterization, Design, Construction, and Monitoring of Bioreactor Landfills. ALT-3. Washington, D.C.: Interstate Technology \& Regulatory Council (ITRC), Alternative Landfill Technologies Team. Interstate Technology \& Regulatory Council [en línea] http://www.itrcweb.org/GuidanceDocuments/ALT-3. pdf 12/02/2014

IWM (1999). The role and operation of the flushing bioreactor. Report of the sustainable Landfill Working Group. Institute of Wastes Management, Northampton (IWM), UK. 63 pp.

Kölsch F. y Ziehmann G. (2004). Landfill stability, risks and challenges. Waste Manage. World. 4, 55-60.

Kraemer T., Kemper C. yAllen J. (2012). Reclaimable Hybrid Bioreactor. Patent No: US008313921B2 Noviembre, 2012 [en línea] https://www.google.com.mx/url?sa=t$\& \mathrm{rct}=\mathrm{j} \& \mathrm{q}=\& \mathrm{esrc}=\mathrm{s} \&$ source $=$ web\&cd $=1 \& \mathrm{cad}=\mathrm{rja} \& u a$ $\mathrm{ct}=8 \& \mathrm{ved}=0$ ahUKEwj005Xn5JXRAhUS4WMKHZY xAtYQFggZMAA\&url=http\%3A\%2F\%2Fpatentimag es.storage.googleapis.com\%2Fpdfs\%2FUS8313921. pdf\&usg=AFQjCNFPV 5 N G 1 KjoD 05 Gx Z13BfI17VCDA\&bvm=bv.142059868,d.amc 06/03/2015

Kumar S., Chiemchaisri C. y Mudhoo A. (2011). Bioreactor landfill technology in municipal solid waste treatment: An overview. Crit. Rev. Biotechnol. 31, 77-97. http://dx.doi.org/10.3109/07388551.2010.492206

Matsufuji Y. (2004). A Road to Semi-aerobic Landfill: Experience of Semi-aerobic Landfills in Japan and Malysia. Memorias. The Third Intercontinental Research Symposium, Hokkaido, Japan. Nov 29-Dic 2, 2004. 1-8.

McCreanor P.T. (1998). Landfill Leachate Recirculation Systems: Mathematical Modeling and Validation. PhD Dissertation. University of Central Florida, Orlando, FL. 231 pp.

MINAM (2008). Guía de diseño, construcción, operación, mantenimiento y cierre de relleno sanitario mecanizado. Elaborado por: Ing. Leandro Sandoval Alvarado Consultor, el Ministerio del Ambiente (MINAM) de Perú y Red de Instituciones Especializadas en Capacitación para la Gestion Integral de los Residuos Solidos [en línea]. https://docs.google.com/ file/d/0B3L0LbO7_abEX3U5QWRUeDY5SGM/view

MINAM (2012). Informe Anual de Residuos Sólidos Municipales y No Municipales en el Perú Gestión 2012. Elaborado por: Ambiente y Desarrollo Sostenible AMBIDES S.A.C. \& Ing. Leandro Sandoval Alvarado. Ministerio del Ambiente (MINAM), Viceministerio de
Gestión Ambiental. [en línea]. http://redrrss.minam. gob.pe/material/20140423145035.pdf 10/05/2014.

O'Brien J. (2009). The Solid Waste Manager's Guide to the Bioreactor Landfill- 2009 Update. Solid Waste Association of North America (SWANA) [en línea]. http:// www.mwcog.org/uploads/committee-documents/ bF5WX15Z20110323110512.pdf 06/12/2009

Olay-Romero E., Hernández-Berriel Ma.C., Díaz-Archundia L.V., de la Rosa-Gómez I., Buenrostro Delgado O. y Márquez-Benavidez L. (2014). Biogas production with two frequencies and rates of leachate recirculation in municipal solid waste. Memorias. The 29th International Conference on Solid Waste Technology and Management. Philadelphia, PA U.S.A. March 30-April 2, 2014. 1146-1155.

OPS-AIDIS-BID (2010). Informe de evaluación regional del manejo de residuos sólidos urbanos en América Latina y El Caribe 2010. Organización Panamericana de la Salud (OPS), Asociación Interamericana de Ingeniería Sanitaria y Ambiental (AIDIS) y Banco Interamericano de Desarrollo (BID). Informe. Washington, DC. 158 pp.

Palma G.J.H., Valenzuela T.P.I. y Espinace A.R.H. (2000). Reducción de los Tiempos de Estabilización en Rellenos Sanitarios Operados con Recirculación de Lixiviados Tratados. Memorias. XXVII Congresso Interamericano de Engenharia Sanitária e Ambiental (AIDIS). Porto Alegre Rio Grande do Sul Brasil. 3 al 8 de Diciembre de 2000. CD-ROM.

PETRAMÁS (2011). De la Basura a la Electricidad: Huaycoloro, la primera experiencia peruana. Seminario Internacional. El Futuro de las Energías Renovables en el Perú. Organismo Superior de la Inversión en Energía y Minería (OSINERGMIN). (Peruanos trabajando por un medio ambiente saludable) [en línea]. http://www. osinerg.gob.pe/newweb/uploads/Publico/SeminarioIntEFERP/Jueves\%206.10.2011/2.\%20De\%20la $\% 20$ basura $\% 20 a \% 201 a \% 20$ ElectricidadJorge $\% 20-\% 20$ Jorge\%20Zegarra.pdf 25/03/2015

PETRAMÁS (2013). Relleno Sanitario Huaycoloro. Petramás S.A.C. Peruanos trabajando por un medio ambiente saludable (Petramás S.A.C.) [en línea]. http:// www.petramas.com/relleno-sanitario-huaycoloro-2/ 25/03/2015.

Pohland F.G. (1975). Sanitary Landfill Stabilization with Leachate Recycle and Residual treatment. Report for United State Environmental Protection Agency (USEPA) Grant No. R-801397. US EPA, National Environmental Research Center, Cincinnati, Ohio. 116 pp.

Pohland F. y Kim J. (1999). In situ anaerobic treatment of leachate in landfill bioreactors. Water Sci. Technol. 40, 203-210. DOI:10.1016/S0273-1223(99)00627-7 
Raju M., Mamat I. B. y Ramli B. (2001). Open Dump to Sanitary Landfill. The Malaysian experience. Memorias. Sardinia 2001. Eight International Waste Management Symposium. Cagliari, Italia, 1-5 Octubre de 2001, 113-120.

Ramly N. H. y Mamat I. B. (2001). Step by step improvement of the Seberang Perai Malaysis Landfill site by using appropriate technology. Memorias. Sardinia 2001, Eight International Waste Management Symposium. Cagliari, Italia, 1-5 Octubre de 2001 121-130.

Reinhart D. R. y Townsend T. G. (1997). Landfill Bioreactor Design and Operation, Lewis Publishers. Boca Ratón, New York, NY. ISBN 1-56670-259-3. 208 pp.

Reinhart D. R., McCreanor P. T. y Townsend T. G. (2002). The bioreactor landfill: Its status and future. Waste Manage. Res. 20, 172-186. DOI: 10.1177/0734242X0202000209

Reinhart D.R., Amini H. y Bolyard S.C. (2012). The Role of Landfills in US Sustainable Waste Management. Environmental Engineer:Applied Research and Practice, American Academy of Environmental Engineers and Scientists, 15:1-6 [en línea] http://www.aaees. org/downloadcenter/EESAppliedResearchandPracticeV15P1.pdf 06/01/2014

Rim-Rukeh A. (2014). An Assessment of the Contribution of Municipal Solid Waste Dump Sites Fire to Atmospheric Pollution. Open J. Atmos. Pollut. 3, 53-60. http://dx.doi.org/10.4236/ojap.2014.33006

Ritzkowski M. y Stegmann R. (2012). Landfill aeration worldwide: Concepts, indications and findings. Waste Manage. 32, 1411-1419. http://dx.doi.org/10.1016/j. wasman.2012.02.020

Rogoff M. (2013). Solid waste recycling and processing: Planning of solid waste recycling facilities and program. Waltham, MA, USA: William Andrew. Burlington: Elsevier Science. ISBN: 978-1-4557-3192-3.

SAIC (2011). Aerobic and Anaerobic Bioreactor Project Protocol. Prepared by Science Applications International Corporation (SAIC). [en linea]. https://www. google.com.mx/url? sa $=$ t\&rct $=\mathrm{j} \& \mathrm{q}=\&$ esrc $=$ s\&sour $\mathrm{ce}=$ web $\& \mathrm{~cd}=1 \& \mathrm{cad}=\mathrm{rja} \& u a c t=8 \& \mathrm{ved}=0$ ahUKEw iqiJCVqZTRAhWb3oMKHc7oCesQFggeMAA\& url $=$ http $\% 3 \mathrm{~A} \% 2 \mathrm{~F} \% 2 \mathrm{Fwww}$.climateactionreserve. org $\% 2$ Fwp-content $\% 2$ Fuploads $\% 2$ F $2009 \% 2$ F03 $\% 2$ FAerobic_Anaerobic_Bioreactor_Issue_Paper_2011. pdf\&usg=AFQjCNERCDe54kS54KTNbĀe3n5AjVYsUg\&bvm=bv.142059868,d.amc 5/04/2015

Sandip M., Kanchan K. y Ashok B. (2012). Enhancement of methane production and bio-stabilisation of municipal solid waste in anaerobic bioreactor landfill. Bioresource Technol. 110, 10-17. http://dx.doi. org/10.1016/j.biortech.2011.12.027
Sang N.N., Soda S., Ishigaki T. y Ike M. (2012). Microorganisms in landfill bioreactors for accelerated stabilization of solid wastes. J. Biosci Bioeng. 114, 243-250. http://dx.doi.org/10.1016/j.jbiosc.2012.04.007

Scharff H. (2007). The Role of Sustainable Landfill In Future Waste Management Systems. Informe de la ISWA, International Solid Waste Association [en línea] https://www.iswa.org/uploads/tx_iswaknowledgebase/618166_Paper.pdf 06/12/2013

SCS ENGINEERS (2011). Protocolo de inspección para la construcción y operación de rellenos sanitarios. Operación de nuevos rellenos sanitarios para desechos sólidos. Archivo No. 16210047.01. Houston Texas. Septiembre 2011. 111 pp.

SEMARNAT (2003). NOM-03-SEMARNAT-2003, Especificaciones de protección ambiental para la selección del sitio, diseño, construcción, operación, monitoreo, clausura y obras complementarias de un sitio de disposición final de residuos sólidos urbanos y de manejo especial. Secretaría de Medio Ambiente y Recursos Naturales. Diario Oficial de la Federación. 12 de septiembre de 1998.

SPREP (2014). Semi-aerobic (Fukuoka) Landfill Management in Samoa. Secretariat of the Pacific Regional Environment Programme (SPREP) [en línea]. https:// www.sprep.org/Case-Studies/semi-aerobic-fukuokalandfill-management-in-samoa 31/06/2014

SPREP-JICA (2010). A Practical Guide to Landfill Management in Pacific Island Countries and Territories. Volume-1, 2nd Edition.: Inland-based waste disposal. Secretariat of the Pacific Regional Environment Programme-Japan International Cooperation Agency (SPREP-JICA). [en línea]. https://wedocs.unep.org/ rest/bitstreams/15642/retrieve 30/04/2014

Sri-Shalini S., Karthikeyana O. P. y Joseph K. (2010). Biological stability of municipal solid waste from simulated landfills under tropical environmental. Bioresource Technol. 101, 845-852. http://dx.doi. org/10.1016/j.biortech.2009.08.104

Thiel R. (2005). Observed Benefits and Problems Associated with Leachate Recirculation. Memorias. Sardinia 2005, Tenth International Waste Management and Landfill Symposium. CISA, Environmental Sanitary Engineering Centre, Cagliari, Italy. 3-7 October 2005. CD-ROM.

Thiel R. y Monte C. (2005). Leachate recirculation and potential concerns on landfill stability. Memorias. 19th Annual GRI Conference, presented at the NAGS 2005/ GRI 19 Conference, Las Vegas, NV, 14-16 December 2005. CD-ROM.

Townsend T., Kumar D. y Ko J. (2008). Bioreactor Landfill Operation:A Guide for Development, Implementation 
and Montoring: version 1.0 (July 1, 2008). Prepared for the Florida Department of Enviroment Protection and The Hinkley Center for Solid and Hazardous Waste Management, Gainesville, FL. [en línea]. http:// nswaienvis.nic.in/Waste_Portal/Technologies/bioreactor_landfill/A_Guide_For_Bioreactor_Landfill_Operation.pdf $10 / 12 / 2013$

Trois C. y Griffith M. R. (2008). Long-term emissions from mechanically biologically treated waste: influence on leachate quality Part II. Water SA. 32, 307314. UN (2011). Avoidance of landfill gas emissions by passive aeration of landfills. United Nations (UN) [en línea]. http://www.scielo.org.za/pdf/wsa/v34n3/08. pdf $25 / 04 / 2014$

UN-HABITAT (2014a). Technical Assistance on Solid Waste Management in Penang Island Through Community Participation. United Nations Human Settlements Programme (UN-HABITAT) [en linea]. http://www.fukuoka.unhabitat.org/projects/malaysia/ detail01_en.html 29/04/2014

UN-HABITAT (2014b). Semi-Aerobic Landfill System (Completed). United Nations Human Settlements Programme (UN-HABITAT). [en linea]. http://www.fukuoka. unhabitat.org/projects/china/detail02_en.html 29/04/2014

UNAM-FE-CECHIMEX (2012). Construyendo ciudades sustentables: experiencias de Pekín y la Ciudad de México. Coordinadores: Sergio Efrén Martínez Rivera y Yolanda Trápaga Delfín. Editado por la Universidad Nacional Autónoma de México, Facultad de Economía y el Centro de Estudios China México. ISBN: 978-60702-2951-0. México. $211 \mathrm{pp}$.

USEPA (2000). Project XL: Yolo County Bioreactor Landfill. United States Environmental Protection Agency (USEPA) [en línea]. https://archive.epa.gov/projectxl/ web/pdf/fact-3.pdf 12/12/2013

USEPA (2003). Biosolids Technology Facts Sheet Use of Landfilling for Biosolids Management. United States Environmental Protection Agency (USEPA). Washington, United States [en línea]. https://nepis. epa.gov/Exe/ZyNET.exe/P10053DP.txt?ZyActionD=Z yDocument $\&$ Client $=E P A \& I n d e x=2000 \% 20 \mathrm{Thru} \% 20$ $2005 \&$ Docs $=\&$ Query $=\&$ Time $=\&$ EndTime $=\&$ Search Method $=1 \&$ TocRestrict $=$ n\&Toc $=\&$ TocEntry $=\& Q F i$ eld $=\& Q$ Field Year $=\& Q$ FieldMonth $=\& Q$ FieldDay $=\&$ UseQField $=\& I n t Q F i e l d O p=0 \&$ ExtQFieldOp $=0 \& X m l$
Query $=\&$ File $=\mathrm{D} \% 3 \mathrm{~A} \% 5$ CZYFILES $\% 5$ CINDEX $\% 20$ DATA\%5C00THRU05\%5CTXT\%5C00000021\%5 CP10053DP.txt\&User $=$ ANONYMOUS\&Password $=\mathrm{a}$ nonymous\&SortMethod $=\mathrm{h} \% 7 \mathrm{C}-\&$ MaximumDocume $\mathrm{nts}=1 \&$ FuzzyDegree $=0 \&$ ImageQuality $=\mathrm{r} 75 \mathrm{~g} 8 / \mathrm{r} 75 \mathrm{~g} 8 /$ $\mathrm{x} 150 \mathrm{y} 150 \mathrm{~g} 16 / \mathrm{i} 425 \&$ Display $=$ hpfr\&DefSeekPage $=\mathrm{x} \&$ SearchBack $=$ ZyActionL\&Back=ZyActionS\&BackDe $\mathrm{sc}=$ Results $\%$ 20page $\&$ MaximumPages $=1 \& Z \mathrm{Z}$ Entry $=1$ 06/02/2009

USEPA (2007). Bioreactor performance. EPA530R-07-007. United States Environmental Protection Agency (USEPA). EPA530-R-07-007 EPA530R-07-007 [en línea]. https://www.epa.gov/sites/ production/files/2016-03/documents/bio-perf.pdf $12 / 12 / 2013$

Viste D. R. (1997). Waste processing and biosolids incorporation to enhance landfill gas. Memorias. Sardinia 97, Sixth International Landfill Symposium, Volume I, S. Margherita di Pula, Cagliari, Italy, 13-17 October 1997, 369-374.

Visvanathan C., Karthikeyan O. P. y Park K. H. (2010). Sustainable landfilling in tropical conditions: comparison between open and closed cell approach. Waste Manage. Res. 29, 386-396. DOI: 10.1177/0734242X10383078

Warith M., Li X. y Jin H. (2005). Bioreactor landfills: State of the art review. Emirates J. Eng. Res. 10, 1-14.

Wong C.L.Y. (2006). Bioreactor technology-The right choice for my landfill. Memorias. SWANA 21st Northwest Regional \& Canadian Solid Waste Symposium. Solid Waste Association of North America (SWANA). Richmond, B.C. April 5-7, 2006. CD-ROM.

Xu Q., Tolaymat T. y Townsend T.G. (2012). Impact of Pressurized Liquids Addition on Landfill Slope Stability. J. Geotech. Geoenviron. Eng. 138, 472-480. http:// dx.doi.org/10.1061/(ASCE)GT.1943-5606.0000609

YCPW (2006). Full scale bioreactor landfill for carbon sequestration and greenhouse emission control. Final Technical Progress Report. D.O.E. Award Number DE-FC26-01NT41152. Yolo County Public Works [en línea]. http://www.yolocounty.org/home/ showdocument?id=1528 06/01/2014.

Zhou Xiao-zhi, Sang Shu-xun y Cao Li-wen. (2012). Municipal solid waste degradation and landfill gas resources characteristics in self-recirculating sequencing batch bioreactor landfill. J. Cent. South Univ. 19, 3551-3557. DOI:10.1007/s11771-012-1442-2 